(Ausgeführt - mit Unterstützung der kaiserlichen Akademie der Wissenschaften aus dem Wedl-Legate - in der biologischen Versuchsanstalt in Wien.)

\title{
Willkürliche Umwandlung von Säugetier-Männchen in Tiere mit aus- geprägt weiblichen Geschlechtscharakteren und weiblicher Psyche.
}

\author{
Eine Untersuchung über die Funktion \\ und Bedeutung der Pubertäts drüsen. \\ Von
}

E. Steinach.

(Hierzu Tafel III-VIII.)

Inhaltsübersicht.

I. Einleitung . . . . . . . . . . . . . . . .

Isolierung und Darstellung der männlichen Pubertätsdrüse durch Hodentransplantation bei unreifen Säugetieren. Funktion der männlichen Pubertätsdrüse . . . . . . . . . . . . . . 72

II. Neue Fragen und Probleme . . . . . . . . . . . . . . 75

III. Operatives Verfahren . . . . . . . . . . . . . . 77

Implantation von Ovarien auf kastrierte Männchen . . . . . . . 77

IV. Ergebnisse . . . . . . . . . . . . . . . . 81

1. Die implantierten Ovarien heilen an und wachsen und reifen im männlichen Körper. . . . . . . . . . . . . . .

2. Die Einwirkungen der männlichen und weiblichen Pubertätsdrüse auf die sekundären Geschlechtscharaktere sind nicht identisch, sondern spezifisch .................

3. Hemmender Einfluss der weiblichen Pubertätsdrüse auf männliche Geschlechtscharaktere. . . . . . . . . . . 86

4. Wachstum von Tube und Uterus im männlichen Körper. . . . . 88

5. Mächtige Entwicklung von Mamma und Mamilla ... . . . . 89

6. Transformierende Wirkungen der weiblichen Pubertätsdrüse . . . 92

A. Finfluss auf Wachstum, Dimensionierung, Gestaltung des Körpers und Skeletts . . . . . . . . . . . . . . . 92

B. Einfluss auf den Haarwuchs. . . . . . . . . . . 101

C. Einfluss auf den Fettansatz . . . . . . . . . . 102 
Seite

7. Umstimmung des psychischen Geschlechtscharakters . . . . . . 102

8. Anhang. . . . . . . . . . . . . . . . . . . . . 105 Das Auftreten von heterologen Geschlechtscharakteren im individuellen Leben . . . . . . . . . . . . . . . . . . . 105

Tafelerklärung . . . . . . . . . . . . . . . . . . 107

\section{Einleitung.}

Isolierung und Darstellung der männlichen Pubertätsdrüse durch Hodentransplantation bei unreifen Säugetieren. Funktion der männlichen Pubertätsdrüse.

Vor einem Jahre habe ich bei jungen Säugern mit Erfolg autoplastische Hodentransplantationen ${ }^{1}$ ) ausgeführt und dadurch den einwandfreien experimentellen Nachweis erbracht, dass die Entwicklung der männlichen Geschlechtsreife unabhängig ist von nervösen, den Keimdrüsen entspringenden Impulsen, und dass sie einzig und allein beherrscht wird von der sekretorischen Funktion der im Hoden weitverzweigten inneren Drüse.

Die Tiere, bei welchen die Hoden in frühester Jugend aus ihrer natürlichen Umgebung losgelöst und auf eine neue fremde Unterlage verpflanzt wurden, sind zu voller Männlichkeit herangewachsen und repräsentieren sich in ihrer ganzen Erscheinung und Mächtigkeit als normale Männchen. Aber auch die speziellen sekundären Geschlechtsmerkmale haben normale Dimension und Gestaltung angenommen. Die Samenblase und Prostata sind zu grossen sekretreichen Drüsen herangereift. Der Penis hat sich zur normalen Länge und Schwellbarkeit entfaltet. Geschlechtstrieb und Potenz sind zur richtigen Zeit erwacht und machen sich mit grosser, oft übernormaler Intensität geltend.

Die eingehenden histologischen Untersuchungen der transplantierten Hoden in verschiedenen Stadien ihres Wachstums und Alters - worüber später noch besonders berichtet werden soll —

1) E. Stein ach, Geschlechtstrieb und echt sekundäre Geschlechtcmerkmale als Folge der innersekretorischen Funktion der Keimdrüsen. I. Präexistente und echt sekundäre Geschlechtsmerkmale. II. Über die Entstehung des Umklammerungsreflexes bei Fröschen. III. Entwicklung der vollen Männlichkeit in funktioneller und somatischer Beziehung bei Sängern als Sonderwirkung des inneren Hodensekretes. Zentralbl. f. Physiol. Bd. 24 S. 551. 1910. - Vgl. ferner: Verhandlungen und Demonstrationen des VIII. internat. PhysiologenKongresses in Wien. Zentralbl. f. Physiol. 1910 Nr. 17 S. 828. 
haben nun das bemerkenswerte Resultat gezeitigt, dass keine einzige Samenzellezur Entwicklung gekommen ist. Die Transplantation hat $z u$ einer"strengen, völlig reinen Isolierung und Darstellung der innersekretorischen Drüse geführt. Innerhalb der Samenkanälchen finden sich ausschliesslich Sertoli'sche Zellen, und ausserhalb derselben sieht man die Leydig'schen Zwischenzellen zu breiten, kompakten Lagern zusammengedrängt, welche an Massigkeit die schmalen und kleinen interstitiellen Inseln des normalen Hodens um vielfaches übertreffen.

Dieses Resultat liess sich durchaus nicht im vorhinein erwarten, denn bei jenen Tieren, bei welchen bisher allein die Anheilung von transplantierten Hoden geglückt war, bei jungen Hähnen, sind gerade die generativen Anteile - sogar bis zur Sekretionsfähigkeit zur Ausreifung gelangt [ $\left.\left.\left.\mathrm{Lod} \mathrm{e}^{1}\right), \mathrm{Fog} \mathrm{e} \mathrm{s}^{2}\right)\right]$, wogégen die sekundären Geschlechtsmerkmale keine vollständige Ausbildung erfahren haben. Allerdings wurde schon früher die Frage experimentell in Angriff genommen, von welchen Gewebsanteilen des Hodens die Erhaltung der Integrität der sekundären Sexualcharaktere bei erwachsenen Säugetieren abhängt. Bouin und $\mathrm{Ancel}^{3}$ ) haben durch Unterbindung der Vasa deferentia, Tandler und Grosz ${ }^{4}$ ) durch Röntgenbestrahlung der Hoden die Ausschaltung der generativen Zellen versucht. Ihre Ergebnisse schienen übereinstimmend für die Unabhängigkeit der sekundären Sexuszeichen von den spermatogenen Elementen zu sprechen. Nun hat sich aber herausgestellt, dass diese Methoden zur Trennung der innersekretorischen und generativen Anteile des Hodens nicht zuverlässig sind. Bouin und Ancel, welchen wir die ersten lehrreichen Aufschlüsse über die Funktion der interstitiellen Zellen verdanken, weisen schon darauf hin, dass noch 6 Monate nach Ligatur des Vas deferens Spermatogonien vorkommen, und diese erst nach ca. 1 Jahre

1) Lode, Zur Transplantation des Hodens bei Hähnen. Sitzungsber. d. Wiener kaiserl. Akad. d. Wissensch. 1895.

2) Foges, Zur Hodentransplantation bei Hähnen. Zentralbl. f. Physiol. 1898 (ferner Pflüger's Arch. 1902).

3) Bouin et Ancel, Verschiedene Abhandlungen in den Comptes rendus de la société de Biologie 1903-1905. Journal de Physiol. et Pathol. général 1904.

4) J. Tandler und S. Grosz, Untersuchungen an Skopzen. Wien. klin. Wochenschr. 1908 Nr. 9. 
völlig verschwunden sind. Ich kann diesen Befund auf Grund eigener Erfahrungen bestätigen und noch hinzufügen, dass diese grosse: Widerstandsfähigkeit mancher Spermatogonien sogar bei Voll-Ligatur, d. h. bei Einschluss der Blutgefässe und Nerven in die Ligatur, zur Beobachtung kommt, und dass in einzelnen Fällen noch Monate nach einfacher Unterbindung des Ductus Regeneration von Samenkanälchen erfolgen kann.

Vor kurzem hat ferner Sim mond $\mathrm{s}^{1}$ ) eine besonders sorgfältige Untersuchung angestellt, um die Wirkung der Röntgenstrahlung auf die Samenzellen des Hodens zu kontrollieren. Es wurde zwar der im allgemeinen elektive, zerstörende Einfluss auf die Spermatogonien konstatiert, aber gleichzeitig hervorgehoben, dass auch nach starker, bzw. nach länger dauernder Bestrahlung vereinzelte unversehrte Samenkanälchen nachuweisen sind; es regenerieren dieselben, und es tritt wieder Spermatogenese auf. Die mit dem Zerfall der spermatogenen Elemente einhergehende Wucherung der Zwischenzellen verliert sich wieder nach Regeneration der Samenzellen. Diese Ergebnisse bestimmten Simmonds, sowohl den Samenzellen als auch den Zwischenzellen innersekretorische Funktion zuzuschreiben, und zwar in dem Sinne, dass normalerweise die Samenzellen für die Integrität der Geschlechtscharaktere Sorge tragen und erst nach Zerstörung der generativen Elemente die sonst spärlichen Zwischenzellen in Wucherung geraten und die Rolle der Samenzellen übernehmen. Diese Auffassung ist nun allerdings nicht haltbar. Meine Versuchsreihen haben gelehrt, dass volle Männlichkeit in somatischer und physiologiseher Beziehung entsteht und fortbesteht, auch wenn sich im Hoden des heranreifenden Tieres keine einzige Samenzelle entwickelt. Aber die Befunde Simmond's erweckten erst recht das dringende Bedürfnis nach einem experimentellen Verfahren, welches ein isoliertes Wachstum der inneren Drüse hervorruft und dadurch gewissermaassen eine Reinkultur von innersekretorischen Zellen unter stets gleichen Bedingungen und bei einer beliebigen Anzahl von Tieren ermöglicht. Diese Aufgabe ist gelöst durch die autoplastische Transplantation der Hoden am jugendlichen Säugetiere.

1) M. Simmonds, Über die Einwirkung von Röntgenstrahlung auf die Hoden. Fortschritte auf dem Gebiet der Röntgenstrahlen Bd. 14. 1909/10. 
Erst die klaren und ubberzeugenden Ergebnisse dieser Methode berechtigten zu dem Schluss ${ }^{1}$, dass die Entwicklung der Pubertät mit allen ihren Erscheinungen in keiner Weise mit den Samenzellen zusammenhängt, sondern ausschliesslich gebunden ist an die normale Tätigkeit der inneren Drüse, und dass diese letztere nicht allein das vollständige Wachstum der Geschlechtsorgane und der anderen somatischen Geschlechtsmerkmale bedingt, sondern auch die spezifische Stimmung, die Erotisierung des Zentralnervensystems verursacht, welcher derGeschlechtstrieb und die ihm dienenden Äusserungen und Reflexfunktionen ihre Entstehung verdanken. Die Abhängigkeit der Pubertätsentwicklung von der inneren Drüse geht - wie die experimentell -erzeugten $Z$ wischenstufen von Männlichkeit zeigen - sogar so weit, dass selbst der Grad der Ausbildung der sekundären Geschlechtscharaktere und der individuellen Unterschiede der sexuellen Veranlagung von der Menge der jeweilig vorhandenen innersekretorischen Elemente bestimmt wird.

Die Wirksamkeit der mit der Keimdrüse vereinten, sogenannten inneren Drüse lässt sich nunmehr scharf definieren: sie besteht in der Entwicklung der Pubertät und Erhaltung der ausgebildeten Geschlechtsreife und Geschlechtscharaktere während des zeugungsfähigen Alters. Die Drüse ist bisher als innerer oder innersekretorischer oder interstitieller Anteil der Keimdrüse beschrieben worden. Nachdem ihre Funktion jetzt von der der Keimdrüse im engeren Sinne unterschieden und genau ermittelt ist, dürfte es sich empfehlen, dieselbe statt der ganz allgemeinen und wenig charakterisierenden Bezeichnung nach ihrer Wirkungssphäre „Pubertätsdrüse" zu benennen, und ich werde von nun ab in diesen und weiteren Studien von der männlichen Pubertätsdrüse und in analogem Sinne von der weiblichen Pubertätsdrüse sprechen.

\section{Neue Fragen und Probleme.}

Auf Grundlage der obigen unzweifelhaft festgestellten Ergebnisse drängte sich mir eine Anzahl neuer Fragen und Probleme auf, deren

1) Steinach, 1. c. S. $16--18$. 
Lösung unsere Kenntnisse von der Pubertätsdrüsenfunktion noch wesentlich zu ergänzen und ganz neue Gesichtspunkte zur Wertung der Bedeutsamkeit der Pubertätsdrüse für die individuelle Entwicklung und für die Geschlechtsrichtung der höheren Organismen $\mathrm{zu}$ schaffen geeignet ist.

Sind die Wirkungen der männlichen und weiblichen Pubertätsdrüse in bezug auf die Ausbildung der Geschlechtscharaktere identisch? Wenn dies der Fall, so müsste unter Anwendung meiner Methode nach homoplastischer Transplantation von Ovarien auf früh kastrierte, jugendliche Männchen die männliche Pubertät mit allen ihren Erscheinungen zur selben Zeit und in annähernd gleichem Grade auftreten wie nach autoplastischer Transplantation der Hoden oder wie bei normal aufwachsenden Männchen. Es würde also die Pubertätsdrüse eines Geschlechts nicht allein die homologen, sondern auch die heterologen sekundären Merkmale hervorrufen können, und jene Gruppe ${ }^{1}$ ) von tierischem und menschlichem Pseudohermaphrodismus, bei welcher ein solches Zusammentreffen zur Beobachtung gelangt, würde in der Identität der Pubertätsdrüsenwirkung ihre Deutung finden, und es müsste dann nur die Seltenheit dieses Vorkommens eine Aufklärung erfahren.

Wenn aber die Versuche gegen die Identität entscheiden und sich eine Spezifität der Wirkung der männlichen und weiblichen Pubertätsdrüse herausstellt, dann muss ermittelt werden, ob sich diese spezifischen Einflüsse lediglich im homologen oder auch im heterologen Organismus manifestieren können.

In letzterem Falle wäre zu untersuchen, ob sich die spezifischen Einflüsse bloss wachstumsfördernd auf vorgebildete Anlagen oder auch transformierend, neugestaltend auf indifferente Anlagen geltend machen, und weiterhin, ob solche Wirkungen der heterologen Pubertätsdrüse sich nur auf somatische oder auch auf psychische und funktionelle sekundäre Gesehlechtsmerkmale erstrecken.

Bei der Verfolgung dieser Punkte stünden wir schliesslich vor dem Problem, willkürlich den Geschlechtscharakter des heranwachsenden Individuums umzustimmen.

Die technische Voraussetzung für die experimentelle Behandlung all dieser Fragen war natürlich die Ausführbarkeit eines erfolgreichen Austausches der Keimdrüsen. Vorversuche überzeugten mich, dass

1) Pseudohermaphrodismus secundarius (Halban). Arch. f. Gynäkol. Bd. 70. 
vorläufig die homoplastische Transplantation der Ovarien auf Männchen die besten Aussichten versprach, und es spitzte sich daher die letzte Frage dahin $\mathrm{zu}$, ob es möglich wäre, junge Männchen durch Ausschaltung der männlichen und Einschaltung der weiblichen Pubertätsdrüsen in Tiere mit vollkommen weiblichen Geschlechtscharakteren und mit weiblicher Psyche umzuwandeln.

\section{Operatives Verfahren.}

Implantation von Ovarien auf kastrierte Männchen.

Die Versuche wurden an Meerschweinchen und Ratten angestellt, bei welchen mir die aus meinen früheren Arbeiten ${ }^{1}$ ) geschöpften praktischen Erfahrungen über das Wachstum und Aussehen der sekundären Geschlechtsmerkmale in den verschiedenen Entwicklungsstadien wesentlich zustatten kamen. Bei Ratten bevorzugte ich Kreuzungsprodukte von weissen Tieren und Abkömmlingen von wilden Ratten, da solchen sicher keine Inzuchtserscheinungen anhaften.

Transplantationen der Ovarien - autoplastisch oder von Weibchen auf Weibchen - sind bekanntlich oft ausgeführt, seitdem ihr Gelingen zuerst von $\mathrm{Halba \textrm {n } ^ { 2 }}{ }^{2}$ ) beschrieben worden war. Hingegen batten Transplantationen der Ovarien von Weibchen auf Männchen bisher keinen Erfolg. Higuchi ${ }^{3}$ ) versuchte sie homoplastisch beim Kaninchen, Schultze ${ }^{4}$ ) heteroplastisch vom Hund auf Kaninchen und vom Meersehweinchen auf Kaninchen mit dem Ergebnis, dass vom 17. Tage ab die Verpflanzungen zugrunde gingen.

Bei meinen Experimenten handelte es sich um den Austausch der Pubertätsdrüsen bei jugendlichen Männchen. Dieselben wurden kastriert und unter einem die Ovarien implantiert. Ich schicke

1) Steinach, Pflüger's Arch. Bd. 56. 1894. III. Mitteilung: Über den Geschlechtstrieb der vor und nach der Pubertät kastrierten Ratten und über das Schicksal der akzessorischen Geschlechtsdrüsen infolge der Kastration S. 333. Ferner: Zentralbl. f. Physiol. Bd. 24 Nr. 13. 1910.

2) Halban, zit. oben und Sitzungsber. d. kais. Akademie in Wien, math.naturw. Klasse Bd. 110. 1901.

3) Higuchi, Arch. f. Gynäkol. Bd. 91. 1910.

4) W. Schultze, Arch. f. Entwicklungsmechanik Bd. 29. 1910. 
diese Bemerkung voraus, weil meine positiven Erfolge an die Kastration geknüpft waren. Bei Kontrollversuchen mit Beibehaltung der Hoden fand keine Anheilung statt, es degenerierten die Transplantate in kürzester Zeit. Als Zeitpunkt für die Operation wählte ich bei Ratten je nach der Wachstumstendenz des betreffenden Wurfes das Alter von 3 oder 4 Wochen, bei Meerschweinchen das Alter von 2 oder 3 Wochen, also ein Jugendstadium, in welchem sich die Tierchen schon selbständig zu ernähren imstande sind, und - worauf es hauptsächlich ankommt - wo erfahrungsgemäss noch keine sichtbare Entfaltung der sekundären Geschlechtsmerkmale begonnen hat, so dass später nicht die Spur einer diesbezüglichen Veränderung der Nachschau entgehen konnte.

Ich operierteserienweise-insbesondere bei den vielköpfigen Würfen der Ratte - d. h. ich operierte mehrere Männchen desselben Wurfes und zog einen normalen oder kastrierten Bruder, eventuell auch eine normale Schwester als Kontrolltiere unter vollständig gleichen äusseren Verhältnissen mit auf. Bei den spärlichen Würfen des Meersch weinchens operierte ich je ein Männchen und zog gewöhnlich einen normalen Bruder mit auf. Die gleichzeitige Aufzucht einwandfreier Kontrolltiere hat sich bei der Beurteilung der Ergebnisse als besonders wertvoll erwiesen.

Der operative Vorgang nahm einen etwas verschiedenen Verlauf, je nachdem die Implantation der Ovarien laparatomisch am peritonealen Überzug der Bauchmuskulatur vollzogen wurde, oder subkutan auf die äussere Fläche dieser Muskulatur.

\section{a) Peritoneale Implantation.}

Das Tier wird in einer Glocke ätherisiert und auf einen aseptischen Halter aus Glas aufgebunden. Die Narkose wird mittels einer tütenförmigen, für Luft durchlässigen Maske vorsichtig aufrecht erhalten. Um die Narkose nicht länger als nötig andauern zu lassen, sind die zur Operation bestimmten Tiere schon am Vortag rasiert. Die glatte Bauchfläche wird nun mit Seife gewaschen und mit warmer Sublimatlösung abgespült. Alle weiteren Manipulationen und die Herrichtung des Instrumentariums geschehen gleichfalls unter aseptischer Vorsehrift.

Ich führe den Schnitt durch die Haut und die Bauchmuskulatur längs der Linea alba, erweitere denselben nach Bedarf mittels feiner Schere nach oben oder unten und lege dann die Bauch- 
höhle frei durch Auseinanderhalten der Wundränder mit kurzen Quetschpinzetten.

Zunächst erfolgt die Kastration. Ich hole den Hoden hervor und zerreisse den bindegewebigen Strang, durch welchen der Nebenhoden am Becken festgehalten ist. Vermöge dieses von mir schon früher verwerteten Kunstgriffes kann ich den Hoden mit Nebenhoden und Samenstrang weit hervorziehen und über den Wundrand legen. Ich wiederhole die Prozedur am zweiten Hoden, bringe die Samenstränge nebeneinander, unterbinde dieselben und exstirpiere mit einem Schnitt beide Hoden. Die Kastration ist auf diese Art in ein paar Minuten vollzogen.

Nun schreite ich zur. Implantation. Ich wähle an der Innenfläche der Bauchmuskulatur beiderseits eine Stelle ans, welche ca. $21 / 2 \mathrm{~cm}$ vom Wundrand und $3-4 \mathrm{~cm}$ vom Becken entfernt ist. Zur Vorbereitung dieses Bodens für die Implantation schaffe ich durch Ritzung des peritonealen Überzugs bzw. der Muskulatur eine leichte flächenhafte Verwundung zu dem Zwecke, erstens eine künstliche starke Hyperämie zu erzeugen, zweitens die Neubildung von Blutgefässen, und dadurch mittelbar die Vaskularisierung des Implantats zu beschleunigen. Diese Maassregel hat sich vorzüglich bewährt und kann als ein das Anwachsen mächtig förderndes Moment angesehen werden.

Im Verlauf der Versuchsreihen hat es sich für die wirksame Verpflanzung der Ovarien am günstigsten erwiesen, wenn das Alter der jungen Weibchen gegenüber dem der Männchen um 2 bis höchstens 4 Wochen im Vorsprunge ist.

Während der letzten Manipulationen sind 1-2 solcher Weibchen vom Gehilfen narkotisiert, aufgebunden und aseptisch hergerichtet. Ich eröffne die Bauchhöhle mit einem Schnitt, ziehe die Ovarien sachte hervor, befreie sie rasch vom überschüssigen Fett, schneide sie mit oder ohne Uterushorn $a b$ und versenke sie in die indes warm gehaltene Bauchhöhle des Männchens. Hierauf hefte ich ein Ovarium nach dem andern mit ein oder zwei Nähten an die zuvor präparierte Stelle an. Die Nähte werden durch Muskeln und Haut geführt und über der Haut geknüpft. Bei Verpflanzung des Ovariums mit anhängender Tube und Uterushorn wird auch das letztere - ohne das Lumen zu verschnüren - durch eine Naht an die Bauchwand fixiert. Endlich schliesse ich die Bauchhöhle mit etwa zehn bis zwölf, zugleich durch Haut- und Muskelränder gehenden 
Nähten und bedecke die Wunde und die Knüpfstellen der Implantationen mit einer dicken Schicht von Xeroformkollodium.

Die operierten Tiere kommen in Glasaquarien, deren Boden mit steriler Watte belegt ist, und verbleiben wenigstens 24 Stunden hindureh nach der Operation in einer Temperatur von $25-30^{\circ} \mathrm{C}$. Dieser Warmhaltung der Gewebe von aussen verdanke ich auch zum Teil den günstigen Ausgang der Implantationen.

Die Tiere (Ratten) - vor der Operatiorr ungefüttert - erhalten 6 Stunden nach derselben gekochte Mileh und am folgenden Tage schon ihr gewöhnliches Futter, d. i. Brot und gekochte Milch. Nach dem viertem Tage ist bei glattem Verlauf die Serie ausser jeder Gefahr und kann zusammen mit den Kontrolltieren in den Käfig gesetzt werden; feine Holzwolle als Streumittel verhütet die mögliche Verunreinigung; in 14 Tagen ist die Verheilung und Vernarbung vollendet.

Die peritoneale Einpflanzung der Ovarien habe ich nur bei Ratten ausgeführt; bei Meerschweinchen aus dem Grunde nicht, weil in diesem jugendlichen Alter die muskulöse Bauchdecke ungemein dünn und rissig ist und daher die Vernähung einer grossen Bauchwunde wegen der sehr massigen Eingeweide einige Schwierigkeit bereitet.

\section{b) Subkutane Implantation.}

Dieses Verfahren kam bei beiderlei Versuchstieren in Anwendung. Die Kastration wurde bei Ratten vom Hodensack aus, bei Meerschweinchen von der Leiste aus vorgenommen. Da in dem jugendlichen Alter der Tiere noch kein Descensus testiculi stattfindet, musste in beiden Fällen der Hoden von aussen her an die Gegend, wo der Einschnitt gemacht wird, bis zur Anspannung der Haut augedrückt werden. Nach Lostrennung der Hoden und Vernähung der Wunden habe ich auf beiden Seiten des Bauches, von der Medianlinie gleich weit entfernt, je eine Hauttasche präpariert, den muskulösen Boden derselben durch Aufritzen leicht lädiert, sodann auf diese wunde Fläche entweder das isolierte Ovarium oder das Ovarium mit Tube und Uterushorn mit feinen Nähten angeheftet und zuletzt die Hauttaschen wieder geschlossen. Im übrigen verliefen Vorbereitung zur Operation und weitere Behandlung der Tiere wie oben geschildert ${ }^{1}$ ).

1) Die Meerschweinchen wurden entsprechend ihrer Gewohnheit mit grünern Futter und Brot aufgezogen. 
Bei der peritonealen Implantation liess sich zwar das Bestehen der Verpflanzung eine Weile durch die Bauchdecken hindurch kontrollieren, aber später verwisehte sich der Eindruck durch die mit dem Wachstum der Tiere einhergehenden Verschiebungen, und es konnte erst durch die Relaparotomie ein sicherer Aufschluss über Gelingen oder Misslingen der Operation gewonnen werden. Hingegen war es bei der subkutanen Implantation ein leichtes, das Schicksal der Ovarien nach Ablauf der anfänglichen Schwellung schon äusserlich, und zwar nach Härte und Umfang, nach Wachstum oder Schwinden der Vorwölbungen richtig zu beurteilen - ganz abgesehen von den sichtbar werdenden Wirkungen der Implantationen.

In etwa $45 \%$ der Versuche waren die Implantationen von Erfolg begleitet. Es versteht sich von selbst, dass mit der Übung, der vielfachen Wiederholung und damit rascheren Durchführung der Operation eine Verdichtung der positiven Fälle eintrat und das perzentuelle Verhältnis bei Anrechnung allein der kunstfertig operierten Tiere eine erhebliche Steigerung erfahren würde.

\section{Frgebnisse.}

1. Die implantierten Ovarien heilen an und wachsen und reifen im männlichen Körper.

An die erste Stelle der Befunde ist die Tatsache zu setzen,. dass die Ovarien im männlichen Körper anheilen, wachsen und funktionsfähig werden.

Die Primärfollikel entwickeln sich zu grossen Follikeln mit normaler Eizelle. Zum Teil gelangen diese Bläschen-Follikel zurvollen Reife, zum Teil sieht man sie geplatzt oder rückgebildet in atretische Follikel oder schon umgewandelt in sogenante Corpora lutea, welche mit typischen Luteinzellen angefüllt sind.

Aus diesem Heilerfolg ergibt sich ein prinzipieller Unterschied zwischen dem Verhalten der weiblichen und männlichen Keimdrüsen bei der Transplantation. Während bei der Verpflanzung des jugendlichen Hodens sich lediglich die Pubertätsdrüse weiter entwickelt, reifen bei der Verpflanzung des Eierstockes auch die generativen 
Gewebe und bleiben jeđenfalls längere Zeit in funktionstüchtigem Zustand bestehen. Eine Isolierung der weiblichen Pubertätsdrüse .erscheint daher, wie später ${ }^{1}$ ) noch erörtert wird, nur in jenen Versuchen durchgeführt, wo durch Zufälle des Heilungsprozesses das Stroma des Transplantates exhalten bleibt, während die generativen Elemente verschwinden. Hingegen gewährt die histologische Untersuchung der Implantate stets einen sehr wichtigen Anhaltspunkt zur Frage, welche innersekretorischen Zellen auf die weibliche Pubertätsentwicklung einen maassgebenden Einfluss ausuben.

In Übereinstimmung mit der Wucherung, welche die Leydig'schen Zellen nach der Verpflanzung des Hodens erfahren, zeigt sich auch eine solche Tendenz bei den grossen protoplasmareichen interstitiellen Zellen im Stroma des transplantierten Eierstockes. Sie schliessen sich zwar nicht zu kompakten Lagern zusammen, wie die Leydig'schen Zellen; ihre Anhäufungen sind mehr oder weniger von gewöhnlichem Bindegewebe durchwachsen, aber ihre Wucherung im ovarialen Stroma ist allgemein und in hohem Grade aufallend. Diese als "weibliche Pubertätszellen" benennbaren Elemente verraten übrigens in bezug auf ihren Durchmesser, ferner auf die Grösse und Färbbarkeit des Kernes eine gewisse Ähnlichkeit mit den männlichen Pubertätszellen und deuten auch durch die Struktur auf ihren sekretorischen Charakter hin. Die implantierten Ovarien bieten daher ein besonders reiches Material zum Studium dieser Elemente, und ich behalte mir die genauere histologische Bearbeitung noch vor.

Wie wir sehen, umfasst das Implantat alle wesentlichen Bestandteile des funktionierenden Ovariums. Der einzige merkliche Unterschied besteht darin, dass dasselbe im Wachstum etwas zurückbleibt und nicht ganz die Grösse erreicht wie beim ausgewachsenen normalen Weibchen.

Über die Dauerhaftigkeit der Verpflanzungen kann ich noch kein abschliessendes Urteil abgeben, da ich die operierten Tiere zum Teil noch länger am Leben erhalten will. Vorläufig verfüge ich über Fälle, bei welchen die Ovarien sich 8-9 Monate auf der neuen Unterlage behauptet haben, was mit Rücksicht auf

1) Vgl. 4. (Wachstum von Tube und Uterus im männlichen Körper). 
die Kurzlebigkeit unserer Versuchstiere bereits als dauernder Erfolg bezeichnet werden kann. Bei einer weiteren Reihe positiv verlaufener Operationen sind die Ovarien nicht in ihrer Gänze zur Anheilung gekommen, aber es ist ein lebensfrischer, oft unscheinbarer Rest zurückgeblieben, welcher vermöge seines Gehaltes an Pubertätszellen analoge Wirkungen vermittelt hat, wie das unversehrte komplete Organ ${ }^{1}$ ).

Die negativ verlaufenen Fälle teilen sich in zwei Gruppen. Bei der einen sind die Einpflanzungen schon innerhalb der ersten drei Wochen durch Vereiterung oder Zerfall zugrunde gegangen; hierher gehören ausnahmslos jene Operationen, bei welchen versuchsweise ältere, z. B. drei- bis fünfmonatliche Ovarien zur Implantation verwendet wurden. Bei der zweiten Gruppe sind die Ovarien erst nach mehrwöchentlichem Bestande resorbiert oder vom Bindegewebe der Umgebung völlig verdrängt. worden.

Dass die Verpflanzung der Eierstöcke auf nicht kastrierte Männchen mit dauerndem Erfolge bisher nicht geglückt ist, habe ich schon oben erwähnt. $\mathrm{Ob}$ es sich hier bloss um Zufälle handelt oder um eine gesetzmässige biologische Gegenwirkung, muss durch eine besondere grössere Versuchsreihe entschieden werden. Zur Lösung der vorliegenden Probleme war nur die dauerhaft wirksame Ovarienimplantation bei kastrierten Männchen absolute Bedingung.

2. Die implantierten Ovarien haben keinen fördernden Einfluss auf das Wachstum der männlichen Merkmale. Die Einwirkungen der männlichen und weiblichen Pubertätsdrüse aufdiesekundären Geschlechtscharaktere sind nicht identisch, sondern spezifisch.

Nachdem sich der Austausch der Pubertätsdrüsen als durchführbar erwiesen hatte, war die Beantwortung der Frage nach der

1) Nach dieser Erfahrung vermute ich, dass die seltenen Fälle von sogenannter Agenesieder Keimdrüsen bei vorhandenen sekundären Geschlechtsmerkmalen, welche für die Unabhängigkeit der letzteren sprechen sollten, ihre Aufklärung eher in der Annahme finden, dass die Keimdrüsen vor der Pubertät vorhanden waren, aber später bis auf leicht zu übersehende Reste, welche die Integrität der Geschlechtscharaktere aufrecht erhalten, zugrunde gegangen sind. 
Identität oder Spezifität der Funktionen nurmehr an die genaue Kenntnis der Wachstumsverhältnisse und Entfaltungszeit der sekundären Geschlechtscharaktere geknüpft. Es sind hiervon zwei Kategorien zu unterscheiden; erstens Charaktere, welche vor oder mit der Pubertät ihre Ausbildung erreichen und zweitens solche, welche erst nach der Pubertät auftreten bzw. zur Vollendung kommen. Wie ich bereits in verschiedenen Arbeiten ${ }^{1}$ ) erörtert habe, zählen bei unseren Versuchstieren zur ersten Kategorie als auffälligste männliche Merkmale das Wachstum und die Ausbildung der Penis-Schwellkörper, Prostata und Samenblasen, zur zweiten Kategorie die grössere Länge, Mächtigkeit und Schwere des Körpers bzw. Skeletts, ferner der stärkere und gröbere Haarwuchs.

Zunächst musste das Augenmerk auf die präpuberalen Charaktere gerichtet werden: Beim drei-bis vierwöchentlichen Rattenmännchen ist der Penis noch ganz unentwickelt, kurz und dünn; an der Spitze tritt der weisse fächerige Penisknorpel frei zutage; eine Eichel ist mangels des Schwellkörpers noch nicht angesetzt. Der Penis des Erwachsenen ist lang, dick, blutgefüllt, leicht vorstülpbar, der Penisknorpel vollkommen verdeckt vom kräftigen Schwellkörper, welcher mit seinem abgestutzten Ende den eichelartigen Abschluss der Rute bildet.

Von der Prostata ist in diesem jugendlichen Alter makroskopisch noch nichts zu erblicken; nach der Pubertät erscheint sie als grosse, lappige, perlgrau durchschimmernde, sekretreiche Drüse.

Die infantilen S a m en blä sch en sind Miniaturgebilde, ca. $4 \mathrm{~mm}$ lang, leer, schlaff. Im reifen Zustande sind sie mächtige Organe, ca. $40 \mathrm{~mm}$ lang, und strotzend mit dem spezifischen gelben, gerinnungsfähigen Sekret gefüllt.

Ähnliche Form- und Grössenunterschiede zeigen die Geschlechtscharaktere der versehiedenen Entwicklungsstadien beim M e e rschweinchen. Liegen solche Extreme vor, so ist die Veränderung im Aussehen der sekundären Merkmale über jeden Zweifel erhaben. Hingegen fällt es schon schwerer, minimale Wachstumszunahmen, wie sie für den Fall eines nur schwachen fördernden Einflusses der Ovarien in Betracht kämen, mit Sicherheit festzustellen. Um in dieser Richtung möglichen Fehlerquellen vorzubeugen, habe ich

1) Ste in ach, zit. oben. 
erstens die Tiere in jenem infantilen Alter operiert, wo reicher Erfahrung gemäss auch bei frühreifen Rassen noch keinerlei Entfaltung der Geschlechtscharaktere stattfindet, zweitens habe ich den Zustand derselben schon bei der ersten Operation protokolliert, um bei der Relaparotomie die Befunde einwandfrei vergleichen zu können, und endlich drittens habe ich - als strengste Kontrolle - bei den Serien meist je ein Tier mit aufgezogen, bei welchem lediglich die Kastration, aber keine Implantation vorgenommen worden war.

$\mathrm{Ob}$ Wirkung eintrat oder nicht, liess sich schon am heranwachsenden Tiere prognostizieren, und zwar aus dem Verbalten des Penis bzw. Schwellkörpers, welcher unter normalen Umständen von der neunten, bei besonders frühreifen Tieren von der siebenten Woche an zu spriessen beginnt, und der sich auch bei meinen Experimenten über Hodentransplantation als besonders empfindliches Reagens für wirksame innere Sekretion erwiesen hat. Die endgültige Entscheidung blieb natürlich der Obduktion oder bei jenen Tieren, welche weiter leben sollten, der Relaparotomie vorbehalten.

Diese Revision der sekundären Geschlechtscharaktere, welche zu verschiedenen Zeiten nach der Pubertät ausgeführt wurde, hat folgenden Befund ergeben: Die Ovarien vermögen das Wachstum der männlichen sekundären Merkmale nicht hervorzurufen. Das Resultat ist negativ, ob die Ovarien im ganz unreifen oder mehr oder weniger reifen Zustand verpflanzt werden, ob sie nur teilweise oder in ihrer Gänze mit allen normalen Gewebselementen zur Anheilung kommen. Die männlichen sekundären Organe bleiben auf der infantilen Stufe stehen wie bei gewöhnlichen Frühkastraten.

Hieraus ergibt sich, dass die Funktion der männlichen und weiblichen Pubertätsdrüsenichtidentisch, sondern spezifisch ist, d. h. dass jede Pubertätsdrüse nur die homologen Merkmale zum Wachstum und zur Ausbildung bringt.

Die relativ seltenen Fälle von sogenanntem sekundären Pseudohermaphrodismus von Tier und Mensch, wobei bloss die Keimdrüsen des einen Geschlechtes vorhanden sind, aber sowohl homologe wie heterologe Charaktere bestehen, lassen sich also nicht, wie angenommen wurde, aus der Identität der Funktion ableiten, sondern sind auf 
anderer Grundlage zu erörtern. Die Spezifität der Pubertäts drüsenfunktion ist es vielmehr, welcher eine geradezu entscheidende Rolle bei der Entwicklung der sekundären Geschlechtscharaktere zufällt. Wäre die fördernde Wirkung der Pubertätsdrüsen nicht spezifisch-homolog, so würde im Embryonalleben nach der frühzeitigen Differenzierung des Keimstockes die Fntwicklung nach beiden Richtungen hin fortschreiten. Es würden die Derivate des Wolff'schen sowie die des Müller'schen Ganges zur Ausbildung gelangen, und es würden dann ausnahmslos hermaphroditisch ausgestaltete Individuen entstehen. Wenn dies aber ausnahmsweise tatsächlich geschieht, so ist das Prinzip der Spezifität der Pubertätsdrüsen nur scheinbar durchbrochen. In diesem Falle dürfte es sich darum handeln, dass die Differenzierung des Keimstockes nicht vollständig, nicht durchgreifend ist, dass im differenzierten Hoden weibliche oder im differenzierten Ovarium männliche Pubertätszellen eingesprengt sind und unter gewissen Bedingungen zu Einfluss gelangen ${ }^{1}$ ).

3. Hemmender Einfluss der weiblichen Pubertätsdrüse auf männliche Geschlechtscharaktere.

Es bleibt das implantierte Ovarium dennoch nicht ohne Einfluss auf die männlichen Sexuszeichen. Derselbe manifestiert sich aber nicht im Sinne einer Förderung, sondern im Gegenteil im Sinne einer Hemmung des Wachstums. Diese Hemmungserscheinung habe ich beim Verfolgen der Entwicklung des Penis und der Schwellkörper ermittelt.

Beim 3-4 wöchigen Rattenmännchen ist der Penis so kurz, dass er sich aus der Vorhaut nicht vorstülpen lässt; nur die Harnröhrenmündung und der Penisknorpel können durch Vordrücken in die Vorhautöffnung sichtbar gemacht werden. Hingegen ist es beim ausgewachsenen Männchen leicht, den dicken, mit dem vollausgebildeten Schwellkörper versehenen Penis in einer Länge von 11-15 $\mathrm{mm}$ aus der weit offenen, schlauchartigen Vorhaut hervorzustülpen. Wird ein infantiles Tier kastriert, so sistiert zwar bekanntlich das Wachstum, aber es sistiert nicht sofort; der Penis

1) Vgl. 8. Anhang. Das Auftreten von heterologen Geschlechtscharakteren im individuellen Leben. 
wächst mit der Harnröhre noch etwas in die Länge, so dass der Stummel beim heranwachsenden Kastraten doch etwa $3 \mathrm{~mm}$ aus der Vorhaut hervorgestülpt werden kann. In diesem Stadium tritt der Stillstand ein. Beim ausgewachsenen oder alten Kastraten findet sich keine Spur weiterer Entwicklung.

Dieses beschränkte und engbegrenzte Wachstum des Penis wird nun bei jenen Tieren, welchen Ovarien mit Erfolg implantiert sind, gehemmt. Der Penis verdient hier nicht mehr seinen Namen und erscheint zu einer Clitoris reduziert. Er ist bei den ausgewachsenen Individuen so kurz, dass er nicht vorgestülpt werden kann; der Schlauch hat sich bis auf eine minimale, eben noch für die Harnentleerung genügende Öffnung zusammengezogen und die charakteristische Vorwölbung, welche derselbe an der Bauchfläche des Männchens bildet, ist gänzlich geschwunden ${ }^{1}$ ). Wenn die implantierten Ovarien nicht anheilen oder im heranreifenden Tiere zugrunde gehen, so fällt die Wachstumshemmung des Penis aus, und derselbe verhält sich wie der des einfachen Kastraten.

Mit der Beobachtung einer solchen Wachstumshemmung ist zum ersten Male der objektive Nachweis erbracht, dass die Pubertätsdrüsen das Wachstum bzw. die Ausbildung von heterologen sekundären Geschlechtscharakteren zu unterdrücken imstande sind. Bekanntlich ist diese Möglichkeit schon theoretisch postuliert worden. Wir werden in einem späteren Abschnitt (6.) noch andere tiefgreifende Wachstumshemmungen seitens des implantierten Ovariums kennen lernen und im Anschlusse hieran auch auf die Frage des ausnahmsweisen Auftretens von heterologen Merkmalen im individuellen Leben zurückkommen.

1) Dass die Hemmung nicht auch bei den andern sekundären Organen (Samenbläschen, Prostata) merkbar wird, darf nicht wundernehmen, da dieselben in dem Alter, wo die Operation stattfindet, noch so rudimentär sind, dass eine Wachstumshemmung oder geringe Rückbildung mit Sicherheit kaum festgestellt werden könnte. - Beim Meerschweinchen lässt sich die Verkürzung des Penis nach der Implantation zwar auch konstatieren, sie erscheint aber nicht so auffallend wie bei der Ratte, weil der Penis schon im infantilen Zustand stärker ausgebildet ist. 
4. Wachstum von Tube und Uterus im männlichen Körper.

Wenn die Tube und ein Stück vom Uterushorn mit dem Ovarium in die Bauchböhle des Männchens verpflanzt werden und das Ovarium anheilt, so macht das letztere seinen spezifischen Einfluss auf die Entfaltung dieser weiblichen Charaktere in der gleichen Weise geltend wie unter normalen Umständen im weiblichen Tiere. Tube und Uterushorn wachsen zu reifen Organen heran und nehmen die charakteristische anatomische Beschaffenbeit und Form an. Gewöhnlich verschliesst sich das Schnittende der Tube bzw. des Uterus, und es sammelt sich in ihnen etwas Serum an, wodurch sie ein pralles und durchscheinendes Aussehen bekommen. Dass das frei in die Bauchhöhle ragende Uterushorn nicht ganz die üppige Fülle erreicht wie am natürlichen Standort, versteht sich bei der ungenügenden, lediglich von der kleinen Implantationsstelle ausgehenden Ernährung von selbst. Ich habe aber in einzelnen Fällen bei der Relaparotomie den ganzen weiblichen Gesehlechtsapparat - Ovarien mit Tube und je einem Uterushorn - so frisch, normal und reif angetroffen, dass sich der Eindruck aufdrängte, es seien alle Bedingungen gegeben, um durch künstliche Befruchtung eine Schwangerschaft einleiten zu können.

Den Gegenversuch stellten jene Implantationen dar, nach welchen die Ovarien nicht Wurzel fassten und der Resorption verfielen. Bei diesen Tieren ergab die Relaparotomie auch völliges Verschwinden von Tube oder Uterus oder Vernichtung derselben bis auf formlose atrophische Rudimente.

Wie schon erwähnt, heilt das Ovarium nicht immer in seiner Gänze an. Oftwird es reduziert, und es bleibt blossein lebensfrischer Rest an der Bauchwand zurück. Auch unter diesen Umständen zeigt sich ein Weiterwachsen von Tube und Uterus. Dieser Befund in Zusammenhang mit der histologischen Untersuchung des ovarialen Restes ist von besonderer Bedeutung. Es findet sich nämlich in solchen Fällen eine Wucherung des Stromagewebes und ein Reichtum von grossen interstitiellen Zellen, aber es finden sich keine Follikel und keine Corpora lutea War schon die Wucherung der interstitiellen Zellen bei den Implantationen ein Fingerzeig für ihre Mitwirkung bei der Pubertätsentwicklung, so erbringt die. sich hier 
ergebende Isolierung der ovarialen $Z$ wischensubstanz rom generativen Gewebe einen direkten Nachweis, dass in Übereinstimmung mit der männlichen Pubertätsdrüse auch im Ovarium es die interstitiellen Zellen sind, welche die weibliche Pubertätsdrüse charakterisieren und das Wachstum und die Ausbildung der sekundären Geschlechtscharaktere hervorrufen.

Das Corpus luteum scheint seine innersekretorischen Funktionen auf anderen Gebieten und hauptsächlich bei der Schwangerschaft geltend zu machen ${ }^{1}$ ).

5. Mächtige Entwicklung von Mamma und Mamilla. (Tafel III und IV.)

Nicht allein auf die mitverpflanzten differenzierten weiblichen Merkmale erstreckt sich die wachstumsfördernde Funktion des Ovariums; es hat auch die Fähigkeit, indifferente Anlagen der Männchen zu differenzieren und zu typischen weiblichen Organen auszugestalten. Es entwickeln sich Brustwarze, Warzenhof und Brustdrüse in der Form und Grösse wie bei normalen Weibchen.

Das infantile Meerschweinchen ${ }^{2}$ ) beiderlei Geschlechts besitzt zwei winzige, aber deutlich ausgeprägte Zitzenanlagen. Die Zitzen erscheinen als etwa $1 \mathrm{~mm}$ lange, dünne, trockene Hautspitzen, welche scharf auf den kleinen, flachen, haarlosen Warzenhof aufgesetzt siıd (Taf. III Fig. 1). Beim heranwachsenden Männchen verändert sich an diesen Rudimenten nichts mehr; die Zitzen werden nur noch welker und dürrer und der Warzenhof verschwindet allmählich unter der dichten Behaarung der unmittelbaren Nachbarschaft.

Beim Weibchen zeigt sich in den ersten Wochen auch keine Vergrösserung der Brustwarzenanlage, aber die Zitzen trocknen nicht ein, sondern werden injiziert und strecken sich. Vom dritten Lebensmonat ab fangen sie an, stärker zu wachsen und sich zu verdicken;

1) Ancel et Bouin, Recherches sur les fonctions du corps jaune gestatif. Journ. de Physiol. et Pathol. géner. t. 13. 1910. - Auf die bezügliche zahlreiche Literatur kann hier nicht näher eingegangen werden.

2) Das Rattenmännchen ist für diesen Punkt der Untersuchung deshalb ungeeignet, weil bei ihm diese Ȧnlagen nicht einmal rudimentär ausgeprägt, bzw. makroskopisch nicht sichtbar sind. 
der Warzenhof breitet sich aus, wölbt sich etwas vor und bleibt haarfrei; langsamen Schrittes geht dann die prägravide Vollendung des Apparates vonstatten.

Derselbe Prozess - nur in beschleunigtem Tempowird beim Männchen durch erfolgreiche Implantation von Ovarien herbeigeführt.

Es wurden zwei- bis dreiwöchige Männchen operiert und gleichaltrige oder um eine Woche ältere Ovarien in der beschriebenen Art subkutan unter die Bauchhaut implantiert. Bei hellfarbigen Exemplaren, bei welchen die Haut pigmentarm ist, liess sich der ganze Hergang und die Reaktion auf das innere Ovarialsekret besonders schön beobachten.

Zuerst entsteht eine heftige Infiltration und Anschwellung der Wundstelle. Die Verpflanzungen fühlen sich hart an. Wenn keine Vereiterung eintritt, melden sich aber bald die günstigen Zeichen des Heilungsverlaufes; es verliert sich die Geschwulst, und die Vorwölbungen werden wieder kleiner und weicher. Dies vollzieht sich innerhalb 14 Tagen. Bis dahin bleiben Warzenhof und Warze biass und unbeteiligt. Nun erfolgt fast plötzlich die Wendung. Die Haut des Warzenhofes wird rot und glänzend. Die Injektion und Hyperämie ergreift die bisher weissen Zitzen, die sich strecken und wie erigiert aussehen. In wenigen Tagen hebt ein rapides Wachstum an, in die Höhe und Dicke, und in wenigen Wochen sind die männlichen Rudimente zu strotzenden weiblichen Organen verwandelt. Der Warzenh of hat sich sehr verbreitert, und die Zitzen erreichen wenigstens die Grösse wie bei vollausgewachsenen, acht- bis zehnmonatigen, jung fräuli chen Weibchen, können aber diesen Grad der Ausbildung noch erheblich überschreiten. Auch die histologische Beschaffenheit stimmt mit der bei normalen Weibchen überein.

Die überraschend mächtige Einwirkung der implantierten Ovarien wird übrigens am besten durch die Abbildung und Photographie der feminierten Männchen veranschaulicht (Taf. III Fig. 2 und Taf. IV).

Wie die mikroskopische Untersuchung lehrt, hält die Entwicklung der Brustdrase gleichen Schritt mit der ihrer Aussenorgane. Während bei infantilen Tieren beiderlei Geschlechtes und auch bei älteren normalen Männchen sich lediglich Ausführungsgänge ohne Endstücke finden, ist durch die Im- 
plantation eine wirkliche Drüse entstanden. Dieselbe setzt sich aus einzelnen Läppchen zusammen, welche durch Bindegewebszüge und breite Fettablagerungen voneinander geschieden sind. Die Endstücke bzw. die Elemente der Lobuli werden von Alveolen oder Acini gebildet, welche von einem einfachen kubischen Epithel ausgekleidet sind. Die Ausführungsgänge münden in weite, mit einem zweischichtigen Zylinderepithel versehene Sin us lactiferi. Der Aufbau dieser Drüsen entspricht demnach vollkommen der Mamma eines reifen, noch unbelegten Weibchens.

Ich verfüge aber über Vergleichspräparate, aus welchen hervor-. geht, dass die Entwicklung der künstlich erzeugten Mamma sogar weiter fortschreiten kann als beim jungfräulichen Weibchen, was sich durch den grossen Reichtum an Alveolen und durch das Vorhandensein einer noch näher zu untersuchenden Sekretion in derselben unzweifelhaft kundgibt ${ }^{1}$ ).

Die absolute Abhängigkeit der Mamma-Entwicklung vom ovarialen Hormon kommt deutlich bei den halbgelungenen Experimenten zum Ausdruck, bei welchen während der Evolution die Implantate der Resorption verfallen. Bei solchen Tieren bleibt die Weiterentwicklung stehen, die angefachte Wachstumstendenz ist machtlos, die sukkulenten Zitzen trocknen wieder ein und repräsentieren nur ein Zwischenstadium in der Reifung des Mamma-Apparates.

Die vorliegenden neuen Tatsachen werfen ein klärendes Licht auf die Frage, obdiesekundären Geschlechtscharaktere imvornhinein in männlicher oder weiblicher Richtung angelegt sind. Die Annahme, dass es sich so verbält, findet sich verbreitet und wurde in letzter Zeit besonders von $\mathrm{Halban}^{2}$ ) ver-

1) Eine genauere Ermittlung dieser quantitativen Verhältnisse bleibt späteren Versuchen vorbehalten. Bei der Intensität der Ovariumwirkung liegt übrigens der Gedanke nahe, das Implantationsverfahren bei normalen weiblichen Tieren praktisch zu verwerten und durch Verstärkung des Wachstums, durch entsprechend grössere Ausbreitung der Milchdrüsenanlage eine günstige Disposition zu schaffen für die gravide Weiterentwicklung und mittelbar für eine wesentlich reichere Milchproduktion. Diese Methode käme zunächst für junge Milchtiere in Betracht. Da auch heteroplastische Transplantation der Ovarialsubstanz gelingt, könnte man es schliesslich auch wagen, die Disposition für die Stillfähigkeit beim Menschen zu verbessern.

2) Arch. f. Gynäkol. Bd. 70 Sep. S. 66. 1903. 
treten; welcher z. B. in bezug auf die Brustdrüse sagt: „Die Mamma ist von Haus aus unter allen Umständen entweder weiblich oder männlich." Diese Vorstellung wird durch obige Befunde widerlegt.

Die Funktion der Pubertätsdrüsen ist überhaupt eine tiefergreifende als bisher vermutet wurde. Dieselbe beschränkt sich nicht auf die Wachstumsförderung, sondern sie bringt auch eine völlige Umgestaltung der Geschlechtscharaktere zustande. Die Umbildung der männlichen Anlage in eine echte Mamma war nur ein Beispiel. Wir werden in den nächstfolgenden Versuchsreihen die transformierende Kraft der weiblichen Pubertätsdrüse bei einem. ganzen Komplex von sekundären Merkmalen betätigt sehen.

6. Transformierende Wirkungen der weiblichen

Pubertätsdrüse.

(Tafel $\mathrm{V}$ bis VIII.)

\section{A. Einfluss auf Wachstum, Dimensionierung, Gestaltung des Körpers und Skeletts ${ }^{1}$ ).}

Das stärkere Wachstum, das grössere Gewicht, die robuste Figur und insbesondere die Mächtigkeit des Skeletts sind ausgesprochen männliche Charaktere, welche erst nach der Pubertät zur vollen Entfaltung kommen.

Bei den von mir meistbenützten Kreuzungsprodukten der weissen und wilden Ratte (Mus decumanus) besteht in den ersten zwei Lebensmonaten zwischen normalen Männchen und Weibchen kein Gewichtsunterschied; während des dritten Monats wird derselbe merklich, aber die auffällige, sich über jeden Zweifel oder Zufall erhebende Differenz macht sich erst gegen Ende des fünften Monats geltend, weshalb ich auch die regelmässige Wägung der operierten und Kontrolltiere erst von diesem Zeitpunkte an aufnahm.

Donaldson ${ }^{2}$ ) hat sich der verdienstlichen Mühe unterzogen, bei einer grösseren Anzahl unter gleichen Verhältnissen aufgezogener weisser Ratten beiderlei Geschlechtes das durchschnittliche Körpergewicht für jede Altersstufe zu bestimmen. Aus seiner Tabelle ergibt sich, dass im zweiten Monat die Weibchen sogar im Vorsprunge

1) Diese Beobachtungen erstrecken sich auf $R$ at te n und $M$ eerschweinchen.

2) Henry H. Donaldson, Boas Memorial Volume New-York 1906. Ferner: The Journal of Comparative Neurology and Psychology vol. 18 no. 4. 1908. 
sind, und dass erst mit Beginn des dritten Monats die Spuren des männlichen Übergewichtes auftreten. Wenn auch die absoluten Werte je nach Rasse oder Aufzucht variieren, so bieten doch die Relationen der Donald son'schen Tabelle ein wertvolles statistisches Material. Hiernach beträgt der Gewichtsunterschied zwischen Männchen und Weibchen im Alter von 150 Tagen durehschnittlich $37 \mathrm{~g}$, im Alter von 365 Tagen $53 \mathrm{~g}$.

Meinen Beobachtungen der Wachstumszunahme lag nicht bloss Gleichaltrigkeit und Gleichrassigkeit zugrunde, sondern es kam zur Erzielung verlässlicher Resultate die besonders strenge Kontrolle in Anwendung, die aus einem Wurfe stammenden Implantations-Tiere nur mit dem mitaufgezogenen normalen Bruder oder mit einem kastrierten Bruder aus demselben Wurfe in Vergleich zu ziehen. Es versteht sich von selbst, dass diese eine Serie bildenden Tiere im gleichen Raum, unter gleicher Pflege und Fütterung herangewachsen waren. Die Wägung wurde ca. alle 8 Tage zur gleichen Tageszeit vorgenommen.

Tabelle I umfasst die Wägungen einer aus vier Tieren bestehenden Serie; drei davon sind Implantations-Tiere; die Wägungen erstrecken sich vom Beginn des sechsten bis zum Beginn des zehnten Monates.

T abelle I (S. 94) zeigt, dass der anfänglich mässige Gewichtsunterschied zwischen dem normalen Männchen und den Implantationstieren (im Durchschnitt $25 \mathrm{~g}$ ) beim Heranwachsen derselben in auffallender Weise zunimmt und anfangs des zehnten Monats im Mittel schon $89 \mathrm{~g}$ beträgt, somit noch mehr, als nach Donaldson der Durchschnittsdifferenz zwischen jährigen Männchen und Weibchen entspricht. Das leichteste unter den Implantations-Tieren und ein gleichaltriges normales Weibchen aus derselben Zucht hatten im Alter von 9 Monaten ungefähr dasselbe Gewicht.

Die systematischen Wägungen von anderen Serien haben nun im Wesen das gleiche Resultat gezeitigt, wie es Tabelle I demonstriert. Die Tendenz des raschen, starken männlichen Wachstums geht einige Zeit nach der Implantation der Ovarien verloren, und die Tendenz des lang. sameren, schwachen weiblichen Wachstums tritt in Erscheinung. 


\section{Tabelle I.}

Betrifft Wägungen von vier schwarzen Ratten aus demselben Wurf; davon sind drei im Alter von ca. 1 Monat operiert, im Alter von 3 Monaten relaparotomiert mit dem Befund der erfolgreichen Ovarienimplantation. Ein Bruder ist als normal mitaufgezogen. Die Wägung beginnt im Alter von 5 Monaten und wird 4 Monate hindurch wöchentlich vorgenommen. Das Photogramm des Normaltieres und eines der Implantations-Tiere ist auf Tafel VI Gruppe 2 reproduziert.

\begin{tabular}{|c|c|c|c|c|}
\hline $\begin{array}{l}\text { Alter } \\
\text { der Tiere } \\
\text { in Tagen }\end{array}$ & $\begin{array}{c}\text { Normales Männ- } \\
\text { chen (Bruder) } \\
\mathrm{g}\end{array}$ & $\begin{array}{c}\text { Implantations- } \\
\text { Tier Nr. I } \\
\text { g }\end{array}$ & $\begin{array}{c}\text { Implantations- } \\
\text { Tier Nr. II } \\
\mathbf{g}\end{array}$ & $\begin{array}{c}\text { Implantations- } \\
\text { Tier Nr. III } \\
\text { g }\end{array}$ \\
\hline $\begin{array}{l}150 \\
158 \\
166 \\
174 \\
182 \\
190 \\
198 \\
206 \\
214 \\
222 \\
230 \\
238 \\
246 \\
254 \\
262 \\
270 \\
278\end{array}$ & $\begin{array}{l}\mathbf{2 6 4} \\
285 \\
316 \\
320 \\
327 \\
326 \\
329 \\
344 \\
346 \\
352 \\
360 \\
367 \\
370 \\
372 \\
385 \\
389 \\
390\end{array}$ & $\begin{array}{l}\mathbf{2 3 6} \\
240 \\
245 \\
255 \\
255 \\
252 \\
255 \\
265 \\
267 \\
275 \\
280 \\
283 \\
280 \\
282 \\
282 \\
288 \\
\mathbf{2 8 5}\end{array}$ & $\begin{array}{l}\mathbf{2 4 0} \\
250 \\
260 \\
270 \\
277 \\
276 \\
282 \\
285 \\
296 \\
300 \\
302 \\
300 \\
300 \\
303 \\
300 \\
300 \\
300\end{array}$ & $\begin{array}{l}\mathbf{2 4 1} \\
\mathbf{2 4 8} \\
245 \\
245 \\
250 \\
258 \\
270 \\
280 \\
288 \\
295 \\
308 \\
308 \\
312 \\
315 \\
320 \\
320 \\
\mathbf{3 2 0}\end{array}$ \\
\hline
\end{tabular}

Gegen diese Deutung könnte der Einspruch erhoben werden, dass das geringere Wachstum, bzw. der grosse Gewichtsunterschied entweder nur von der gleichzeitig mit der Verpflanzung erfolgten Kastration herrühre oder überbaupt durch die verschiedenen operativen Eingriffe bedingt sei.

Die Kastration als ursächliches Moment wurde ausgeschlossen durch Kontrollwägungen bei normalen und kastrierten Männchen aus demselben Wurfe. Es hat sich herausgestellt, dass bei annähernd gleichem Gewicht der Brüder zu Beginn des vierten Monats im Verlaufe der nächsten 4 Monate eine Differenz zugunsten des normalen von höchstens $15 \mathrm{~g}$ entstand, dass sich also bei Rattenmännchen die Wachstumskurve der Normalen und Kastraten vollkommen ähnlich verbält, In bezug auf diesen Befund kann ich mich übrigens auch auf eine eigens diesem Vergleich gewidmete Arbeit von Stotsenburg ${ }^{1}$ ) berufen, welcher diese Verhältnisse an

1) On the growth of the Albino Rat after Castration (aus dem Wistar Institut). The Anatomical Record vol. 3. 1909 
einem grösseren Material studiert hat und $\mathrm{zu}$ demselben Resultat gelangt ist.

Dass die Gewichtszunahne weder durch die Kastration noch durch die übrigen chirurgischen Insulte dauernd be einträchtigt war, habe ich dadurch einwandfrei bewiesen, dass ich Tiere desselben Wurfes verglich, welche allen Eingriffen in der gleichen Weise unterzogen wurden, von denen aber einige wegen Misslingens der Ovarium-Anheilung nichts anderes als Kastraten geblieben waren. Aus diesen Beobachtungsreihen soll Tabelle II ein Beispiel für die Ratten, Tabelle III ein solches für die Meerschweinchen vorführen ${ }^{1}$ ).

\section{Tabelle II.}

Betrifft Wägungen von zwei Ratten desselben Wurfes; dieselben sind im Alter von 4 Wochen operiert, die eine mit positivem und dauerndem Erfolg, die zweite mit negativem Erfolg (typischer Kastrat geblieben). Die Wägung beginnt im Alter von 5 Monaten und wird durch ca. 4 Monate wöchentlich fortgesetzt.

\begin{tabular}{c|c|c|c|c|c}
\hline $\begin{array}{c}\text { Alter } \\
\text { der Tiere } \\
\text { in Tagen }\end{array}$ & $\begin{array}{c}\text { Kastrat } \\
\text { (Bruder) } \\
\mathrm{g}\end{array}$ & $\begin{array}{c}\text { Im- } \\
\text { plantations- } \\
\text { Tier } \\
\mathrm{g}\end{array}$ & $\begin{array}{c}\text { Alter } \\
\text { der Tiere } \\
\text { in Tagen }\end{array}$ & $\begin{array}{c}\text { Kastrat } \\
\text { (Bruder) } \\
\mathrm{g}\end{array}$ & $\begin{array}{c}\text { Im- } \\
\text { plantations- } \\
\text { Tier } \\
\mathrm{g}\end{array}$ \\
\hline & $\mathbf{2 9 4}$ & $\mathbf{1 8 3}$ & 222 & 278 & 212 \\
150 & 233 & $\mathbf{1 8 5}$ & 230 & 290 & 218 \\
158 & 250 & 190 & 238 & 295 & 220 \\
166 & 257 & 198 & 246 & 292 & 220 \\
174 & 257 & 195 & 254 & 295 & 218 \\
190 & 260 & 197 & 262 & 295 & 220 \\
198 & 267 & 202 & 270 & 305 & 219 \\
206 & 270 & 205 & 278 & $\mathbf{3 0 5}$ & $\mathbf{2 1 9}$ \\
214 & 270 & 208 & & & \\
\end{tabular}

Nach Tabelle II beträgt der Gewichtsunterschied $z$ wischen dem Kastraten und dem feminierten Bruder im Alter von 278 Tagen $86 \mathrm{~g}$, also ungefähr ebensoviel wie zwischen dem normalen Männchen und den feminierten Tieren (Tabelle I).

Die diesbezüglichen Erhebungen bei Meerschweinchen (Tabelle III, S. 96) decken sich im Wesen vollständig mit denen der Ratten. Die Gewichtsdifferenz ist hier schon nach 3 Monaten beträchtlich und erreicht im sechsten Monat die respektable Höhe von $200 \mathrm{~g}$.

1) Siehe auch Tabelle VI, betreffend den Vergleich der Körperdimensionen von Kastrat und Implantations-Tier. 
Tabelle III.

Betrifft Wägungen von zwei Meerschweinchen desselben Wurfes. Dieselben sind im Alter von 3 Monaten operiert, das eine mit positivem und dauerndem Erfolg (vgl. Tafel IV), das zweite mit negativem Erfolg (typischer Kastrat geblieben). Die Wägung beginnt im Alter von $3^{1 / 2}$ Monaten und wird ca. 4 Monate hindurch wöchentlich fortgesetzt.

\begin{tabular}{|c|c|c|c|c|c|}
\hline $\begin{array}{c}\text { Alter } \\
\text { der Tiere } \\
\text { in Tagen }\end{array}$ & $\begin{array}{c}\text { Kastrat } \\
\text { (Bruder) } \\
\mathrm{g}\end{array}$ & $\begin{array}{c}\text { Im- } \\
\text { plantations- } \\
\text { Tier } \\
\mathrm{g}\end{array}$ & $\begin{array}{c}\text { Alter } \\
\text { der Tiere } \\
\text { in Tagen }\end{array}$ & $\begin{array}{c}\text { Kastrat } \\
\text { (Bruder) } \\
\mathrm{g}\end{array}$ & $\begin{array}{l}\text { Im- } \\
\text { plantations- } \\
\text { Tier } \\
\text { g }\end{array}$ \\
\hline $\begin{array}{l}105 \\
113 \\
121 \\
129 \\
137 \\
145 \\
153 \\
161 \\
169\end{array}$ & $\begin{array}{l}\mathbf{5 1 2} \\
537 \\
550 \\
556 \\
593 \\
609 \\
637 \\
676 \\
675\end{array}$ & $\begin{array}{l}\mathbf{4 0 8} \\
428 \\
450 \\
457 \\
466 \\
475 \\
485 \\
490 \\
485\end{array}$ & $\begin{array}{l}177 \\
185 \\
193 \\
201 \\
209 \\
217 \\
225 \\
233\end{array}$ & $\begin{array}{l}683 \\
685 \\
690 \\
685 \\
686 \\
685 \\
688 \\
\mathbf{6 8 8}\end{array}$ & $\begin{array}{l}490 \\
486 \\
490 \\
485 \\
488 \\
485 \\
485 \\
\mathbf{4 8 6}\end{array}$ \\
\hline
\end{tabular}

Bei Meerschweinchen habe ich auch die sogenannten halbgelungenen Fälle und ibre Kontrolltiere der systematischen Wägung unterworfen und dadurch Gelegenheit gefunden, den hemmenden Einfluss der Ovarien in besonders drastischer Weise am Werk zu sehen. Wie schon oben erwähnt, ereignet es sich manchmal, dass das implantierte Ovarium alle Anstalten zur Anheilung macht und bereits deutliche Zeichen seiner Wirksamkeit gibt - Injektion und Vergrösserung der Zitzen, Zurückbleiben des Körperwachstums -, und dass es dann aus unbekannten Gründen doch resorbiert wird und allmählich unter der Haut verschwindet. Diesem Ausfall der Ovarien folgt nun sofort auch die Unterbrechung der Funktion - die Weiterentwicklung der Zitzen hört auf, und das Körpergewicht schnellt dermassen in die Höhe, dass es in wenigen Wochen wieder dem des normalen. Kontrolltieres entspricht.

Nach dem Ergebnis aller dieser Beobachtungen steht es ausser Zweifel, dass die bedeutsame Herabminderung bzw. Hemmung des männlichen Körperwachstums ausschliesslich durch die innersekretorische Tätigkeit des implantierten Ovariums verursacht ist und überaus treffend die erwirkte Femination zum Ausdruck bringt. 
Im Einklang mit den Wägungsresultaten befinden sich die Unterschiede in den Dimensionen der Statur und einzelner Körperteile. Schon bei flüchtigem Vergleich fallen die Implantations-Tiere auf durch die Schmächtigkeit des Kopfes, welche sich besonders in der kleineren Distanz der Ohren und in der merklichen Kürze ausprägt, ferner durch die schlankere Gestalt, und endlich durch die erheblich geringere Länge des Gesamtkörpers das sind aber gerade jene Dimensionen und Formen, durch welche sich die normalen Weibchen auszeichnen. Um hierfür ein Beispiel zu bringen, habe ich betreffende Messungen. an ausgewachsenen älteren Normaltieren (Ratten) in Tabelle IV ausgeführt.

\section{Tabelle IV.}

Körperdimensionen von normalen Männchen und Weibchen; fünf Ratten aus einem Wurf; 15 Monate alt.

\begin{tabular}{l|c|c|c}
\hline Geschlecht der Tiere & $\begin{array}{c}\text { Kopfbreite } \\
\mathrm{mm}\end{array}$ & $\begin{array}{c}\text { Kopflänge } \\
\mathrm{mm}\end{array}$ & $\begin{array}{c}\text { Körperlänge } \\
\mathrm{mm}\end{array}$ \\
\hline Männchen . . . . . . & 27 & 55 & 283 \\
Weibchen I . . . . . & 22 & 50,5 & 208 \\
Weibchen II & 22 & 49,5 & 205 \\
Weibchen III . . . . . & 21,5 & 50 & 205 \\
Weibchen IV. . . . & 21,5 & 49,5 & 205
\end{tabular}

In Tabelle $\mathrm{V}$ sind nun die Messungen einer Implantationsserie (Ratten) und ihrer Kontrolltiere zusammengestellt. Für die wiederholten Prozeduren wurden die Tiere leicht ätherisiert. Kopflänge und Kopfbreite wurden mittels feiner Schubleere bestimmt, und zwar die Kopfbreite durch die Ohrdistanz, die Kopflänge. durch die Entfernung vom Tuber occipitale bis zur Nasenspitze; die Körperlänge wurde gemessen von der Nasenspitze bis zum Schwanzansatz. Bei Aufnahme der Körperlänge wurden die Tiere nicht gestreckt, sondern in natürlicher Verfassung auf die Tischplatte gelegt. Der Brustumfung wurde mittels Bandmaass dicht hinter den vorderen Extremitäten gemessen.

Tabelle VI enthält korrespondierende Messungen an einer Serie von Meerschweinchen. 


\section{Tabelle V.}

Körperdimensionen von Implantations-Tieren und normalen Kontrolltieren. Fünf Ratten; 9 Monate alt (ein normales Männchen und drei Implantations-Tiere aus demselben Wurf; vgl. Tabelle I); ferner ein gleichaltriges normales Weibchen aus derselben Zucht.

\begin{tabular}{l|c|c|c|c}
\hline \hline Geschlecht & $\begin{array}{c}\text { Kopfbreite } \\
\mathrm{mm}\end{array}$ & $\begin{array}{c}\text { Kopflänge } \\
\mathrm{mm}\end{array}$ & $\begin{array}{c}\text { Brustumfang } \\
\mathrm{mm}\end{array}$ & $\begin{array}{c}\text { Körperlänge } \\
\mathrm{mm}\end{array}$ \\
\hline Männchen . . . . . . & 27 & 55 & 155 & 250 \\
Implantations-Tier I . . & 22 & 51,5 & 135 & 225 \\
Implantations-Tier II . . & 22,5 & 52 & 135 & 230 \\
Implantations-Tier III. . & 22 & 52 & 135 & 230 \\
Weibchen. . . . . . . & 22 & 52 & 130 & 225 \\
\hline
\end{tabular}

Tabelle VI.

Körperdimensionen von drei Me ers $\mathrm{ch}$ we in chen; 8 Monate alt (Implantationstier und männlicher Kastrat aus demselben Wurf; vgl. Tabelle III; ferner ein gleichaltriges normales Männchen aus derselben Zucht). Die Kopfbreite wurde an der Jochbogendistanz gemessen.

\begin{tabular}{l|c|c|c|c}
\hline Geschlecht & $\begin{array}{c}\text { Kopf breite } \\
\mathrm{mm}\end{array}$ & $\begin{array}{c}\text { Kopflänge } \\
\mathbf{m m}\end{array}$ & $\begin{array}{c}\text { Brustumfang } \\
\mathbf{m m}\end{array}$ & $\begin{array}{c}\text { Körperlänge } \\
\mathrm{mm}\end{array}$ \\
\hline Männchen . . . . . & 49 & 76 & 212 & 278 \\
Männlicher Kastrat. . . & 43 & 76 & 210 & 275 \\
Implantations-Tier . . . & 40 & 69 & 172 & 245
\end{tabular}

Aus den letzten drei Tabellen ist ersichtlich, dass das Verhältnis der Körperdimensionen zwischen den Implantations-Tieren und Männchen - abgesehen von geringfügigen Varianten - eine überraschende Kongruenz aufweist mit dem Verhältnis der Körperdimensionen zwischen normalen Weibchen und Männchen. Die Tiere mit implantierten Ovarien haben in der Tat die Dimensionen und Formen von Weibchen angenommen.

Gingen die Ovarien während dieser ihrer transformierenden Funktion zugrunde, so kam wieder die ursprünglich männliche Richtung im Wachstum und in der Dimensionierung des Körpers zum Durchbruch und zur schliesslichen Ausbildung.

Wenn auch die vergleichende Wägung und Abmessung der Tiere den transformierenden Einfluss der weiblichen Pubertätsdrüse auf Körperwachstum und Statur in überzeugender Weise darlegten, so war es mir doch darum zu tun, einige exakte Daten über 
die umgestaltende Wirkung speziell am Knochensystem zu ermitteln. Diese Absicht liess sich an den lebenden Ratten durch Röntgen-Untersuchung durchführen. Die Aufnahmen der verschiedenen Serien wurden im Röntgeninstitut des Wiener allgemeinen Krankenhauses gemacht. Es ist mir eine angenehme Pflicht, dem Vorstande desselben, Dozenten Dr. Holzknecht, für seine persönliche Bemühung auch an dieser Stelle verbindlichen Dank auszusprechen.

Ein Tier nach dem anderen wurde narkotisiert, auf die Platte gelegt, mit zwei Stoffstreifen leicht an dieselbe angedrückt und je nach der Qualität der Lampe einige Sekunden bestrahlt. Die Entfernung der Lampe vom Tier blieb bei allen Aufnahmen konstant.

Die Tiere wurden zuerst im Alter von ca. 6 Monaten und in einem Abstand von 2-3 Monaten ein zweites Mal aufgenommen. Durch Vergleich der früheren und späteren Röntgenbilder gewinnt man einen überaus klaren Einblick in den immer schärfer auftretenden Unterschied des Skelettwachstums zwischen den normalen und feminierten Männchen. Für die vorliegende Abhandlung war ich genötigt, aus den Reproduktionen eine enge Auswahl zu treffen, und musste mich daher darauf beschränken, in Tafel V-VII einige Stichproben aus den aufgenommenen Serien vorzuführen und zwar:

Gruppe 1 (Taf. V): Bruder und Schwester aus einem 15 Monate alten Wurf. Diese Parallele soll die äusserste Differenz zwisehen der Grösse und Mächtigkeit des männlichen und weiblichen Skelettes bei normalen, vollständig ausgewachsenen Tieren zur Anschauung bringen.

Gruppe 2 (Taf. VI): Normales Männchen und feminierter Bruder aus einem 9 Monate alten Wurfe.

Gruppe 3 (Taf. VII): Männlicher Frühkastrat und feminierter Bruder aus einem 8 Monate alten Wurf.

Die vorliegenden Röntgenbilder sind zwar dem Studium bzw. der Darstellung des Knochensystems gewidmet; sie demonstrieren aber gleichzeitig auch den Kontrast zwischen den feminierten Tieren und Männchen in bezug auf die Mächtigkeit und Gestaltung der Weichteile.

Die Abmessungen der Länge einzelner Knochen und Skelettteile auf Grund der Originalaufnahmen sind gruppenweise in T abelle VII eingetragen. 


\section{Tabelle VII.}

Vergleichende Knochenmessungen ${ }^{1}$ ) an Ratten nach unter konstanten Bedingungen aufgenommenen Röntgenbildern.

Gruppe 1: normales Männchen und normale Schwester aus einem 15 Monate alten Wurf.

Gruppe 2: normales Männchen und feminierter Bruder aus einem 9 Monate alten Wurf.

Gruppe 3: männlicher Frübkastrat und feminierter Bruder aus einem 8 Monate alten Wurf.

\begin{tabular}{|c|c|c|c|c|c|c|c|c|c|}
\hline \multirow{2}{*}{$\begin{array}{l}\text { Bezeichnung des } \\
\text { Knochens bzw. der } \\
\text { Distanz }\end{array}$} & \multicolumn{3}{|c|}{ Gruppe 1} & \multicolumn{3}{|c|}{ Gruppe 2} & \multicolumn{3}{|c|}{ Gruppe 3} \\
\hline & $\begin{array}{c}\delta \\
\text { normal } \\
\text { mm }\end{array}$ & $\begin{array}{c}\text { 9 } \\
\text { normal } \\
\text { mm }\end{array}$ & $\begin{array}{l}\text { Diffe- } \\
\text { renz } \\
\mathrm{mm}\end{array}$ & $\begin{array}{c}\delta \\
\text { normal } \\
\text { mm }\end{array} \mid$ & $\begin{array}{c}\text { femi- } \\
\text { niert } \\
\text { mm }\end{array}$ & $\begin{array}{l}\text { Diffe- } \\
\text { renz } \\
\mathrm{mm}\end{array}$ & $\begin{array}{c}\text { đ } \\
\text { rastrat } \\
\text { mm }\end{array}$ & $\begin{array}{c}\text { femi- } \\
\text { niert } \\
\text { mm }\end{array}$ & $\begin{array}{l}\text { Diffe- } \\
\text { renz } \\
\text { mm }\end{array}$ \\
\hline 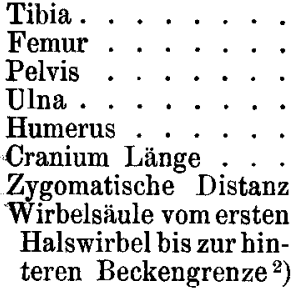 & $\begin{array}{l}42,5 \\
36,5 \\
48 \\
38 \\
30,5 \\
45 \\
24\end{array}$ & $\begin{array}{l}\mathbf{3 9} \\
\mathbf{3 3}, \mathbf{5} \\
\mathbf{4 3} \\
\mathbf{3 1} \\
\mathbf{2 8} \\
\mathbf{4 4} \\
\mathbf{2 3}\end{array}$ & $\begin{array}{l}3,5 \\
3 \\
5 \\
2 \\
2,5 \\
1 \\
1\end{array}$ & $\begin{array}{l}45 \\
36 \\
47 \\
33 \\
31 \\
45 \\
24\end{array}$ & $\begin{array}{l}\mathbf{4 0} \\
\mathbf{3 3} \\
\mathbf{4 2} \\
\mathbf{3 1} \\
\mathbf{2 8} \\
\mathbf{4 2} \\
\mathbf{2 9 , 5}\end{array}$ & $\begin{array}{l}5 \\
3 \\
5 \\
2 \\
3 \\
3 \\
1,5\end{array}$ & $\begin{array}{l}41 \\
33,5 \\
42 \\
32 \\
28 \\
44,5 \\
22,5\end{array}$ & $\begin{array}{l}38 \\
31 \\
38 \\
29 \\
26 \\
42 \\
21,5\end{array}$ & $\begin{array}{l}3 \\
2,5 \\
4 \\
3 \\
2 \\
2,5 \\
1\end{array}$ \\
\hline
\end{tabular}

Wie die in Tafel III-V und Tabelle VII verwerteten Beispiele verhalten sich sämtliche Tiere, bei welchen die Ovarien mit danerndem Erfolge angeheilt waren. Am augenfälligsten sind die Untersehiede betreffs Länge und Dicke an den langen Röhrenknochen, am Becken und an der Wirbelsäule. Berücksichtigt man die völlig ausgewachsenen Tiere (Gruppe 1 und 2), so entspricht die Differenz zwischen feminierten Tieren und Kontrollmännchen jener zwischen normalen Weibchen und Kontrollmännchen; sie ist für einzelne Knochen sogar grösser. Vergleicht man bei denselben Gruppen das feminierte Männchen mit dem normalen Weibchen, so ergibt sich eine überraschende Ähnlichkeit der Knochenmasse. Die genauere Untersuchung des Knochensystems bekräftigt somit die Ergebnisse der oben erörterten gröberen Messungen und zeigt zugleich,

1) Bruchteile von Millimetern sind nur ausnahmsweise berücksichtigt.

2) Wegen der jeweilig etwas verschiedenen Krümmung der Wirbelsäule wurde für diese Distanz das Mittel aus mehreren gleichzeitigen Aufnahmen gezogen. 
wie bei der Femination des zur Männlichkeit bestimmten Individuums die Hemmung des Körperwachstums und die Transformierung der Gestalt sowie der anderen sekundären Merkmale zusammenwirken.

Wenn man der Auffassung Tandler's ${ }^{1}$ ) Rechnung trägt, dass die sekundären Geschlechtscharaktere ursprünglich Speziescharaktere waren und erst nach und nach unter die Herrschaft des Genitales gekommen sind, so muss der Einfluss der Pubertätsdrüse, welcher die Geschlechtscharaktere mit einem Schlage zu wandeln vermag, als eine gewaltige Leistung imponieren.

Um den Gesamteindruck, welchen die feminierten Tiere in Grösse und Figur gegenüber den Kontrolltieren erwecken, einigermassen wiederzugeben, habe ich die Photographien von einem Meerschweinchenpare und zwei Rattenpaaren a uf Tafel VI zur Reproduktion gebracht; je ein Paar ist auf derselben Platte aufgenommen. Der Kontrast im Aussehen der feminierten und normalen Männchen wird durch Nebeneinanderstellung von normalen Weibchen und Männchen in keiner Richtung übertroffen.

\section{B. Einfluss auf den Haarwuchs.}

Bei den zahmen Ratten gibt es im jugendlichen Alter keine besonderen Kennzeichen in der Behaarung beider Geschlechter. Erst vom sechsten Monat $a b$ beginnt eine Veränderung sich bemerkbar zu machen. Die Haare der Männchen werden gröber und länger, die der Weibchen bleiben kürzer, feiner und weicher. Den Höhepunkt in der Differenzierung des Haarkleides erreichen die Tiere aber erst im zehnten oder zwölften Monate. In diesem Zeitpunkt ist das rauhe, oft struppige Haar der Männchen vom geschmeidigen Haar der Weibehen scharf unterschieden.

In derselben Altersstufe, wo sich der weibliche Charakter in der Behaarung bei den normalen Weibchen einstellt, äussert er sich auch beiden feminierten Männchen. Der Kontrast ist ebenso leicht sichtbar wie fühlbar. Streift man den Ratten über den Rücken, so greift sich das Haar der feminierten Männchen glatt und seiden-

1) Zitiert oben. 
a rtig, das der Kontrolltiere (normale und kasstrierte Männchen) derb und echt fellartig an. Durch dieses feine, sich anschmiegende Haarkleid wird der weiblich-grazile Eindruck, den die feminierten Tiere machen, noch erhöht. Je besser die Implantation gelungen, d. h. je mehr ovariale Substanz in Funktion getreten ist, desto deutlicher kommt neben den übrigen weiblichen Geschlechtsmerkmalen auch die weibliche Behaarung zum Ausdruck.

\section{Einfluss auf den Fettansatz.}

Zur Zeit, wo unter normalen Umständen die Pubertät anfängt, lässt sich bei den feminierten Rattenmännchen eine Erscheinung wahrnehmen, welche die Mitte hält zwischen somatischer Transformierung und funktioneller Umstimmung. Es handelt sich um eine ganz charakteristische Fettbildung, welche sich als ausgesprochenes sekundäres weibliches Sexuszeichen erweist. Beim normalen reifen Weibchen begleiten massige Stränge von Fett beiderseits das Uterushorn und füllen in der Gegend des vereinigten Uterus das Becken aus. Bei der Relaparotomie der feminierten Tiere hat sich nun gezeigt, dass in allen Fällen, wo die Ovarien gut angeheilt waren, neben den anderen sekundären Wirkungen auch diese typischen, bei Eröffnung der Bauchhöhle sich vordrängenden Fettlager entstanden sind. In den Fällen, wo die Ovarieu nicht angewachsen sind oder resorbiert worden waren, haben sich auch diese Fettlager nicht entwickelt, ebenso wie solche fehlen bei männlichen Kastraten oder bei normalen Männchen. Dieser speziell weibliche Fettansatz tritt am auffälligsten in Erscheinung in den ersten Monaten der Reife, also in dem Alter, wo die übrigen Organe wie Darmtrakt und Muskulatur noch nicht verfettet sind und die lokalisierten Fettmassen von der Umgebung noch abstechen.

7. Umstimmung des psychischen Geschlechtscharakters.

Bei den feminierten Männchen - Ratten wie Meerschweinchen - entsteht zur Pubertätszeit keine Spur eines männlichen Geschlechtstriebes. Auch wenn man brünstige Weibchen in ihr 
Abteil bringt, verraten sie nichts von Interesse oder Erregung; nach Befriedigung der ersten Neugierde, die jedem Neuling in gleicher Weise entgegengebracht wird, herrseht bald wieder Ruhe und gänzliche Indolenz. Dieses Ausbleiben des männlichen Triebes war zu erwarten und entspricht vollkommen der "Spezifität der Pubertätsdrüsenfunktion". Wir haben oben (S. 85) auseinandergesetzt, dass das implantierte Ovarium keine fördernde Wirkung auf die männlichen sekundären Merkmale auszuüben vermag, und wir sehen nunmehr, dass dieses Prinzip auch in bezug auf den psychischen Geschlechtscharakter gewahrt ist.

Hingegen macht sich ein analoger Vorgang geltend wie bei der Transformierung der somatischen Sexuszeichen; es kommt zur Umstimmung der sexuellen Disposition, zur Entstehung von weiblichem Reiz, kurz zur Erotisierung des Zentralnervensystems ${ }^{1}$ ) in weiblicher Richtung. Die objektiven Symptome dieser Umstimmung äussern sich durch Reaktionen, welche beim Zusammentreffen von feminierten Tieren und normalen Männchen gegenseitig ausgelöst werden.

1. Die feminierten Ratten zeigen wie die normalen Weibchen den "Schwanzreflex", d. i. das oft senkrechte Hochheben und dauernde Hochhalten des Schwanzes während der Verfolgung seitens der Männchen. Dieser Reflext on us dient dem treibenden Männchen, das sich hauptsächlich durch den Geruch orientiert, zum leichten Erkennen des Geschlechtes und insbesondere der Brünstigkeit. Beim Männchen oder Kastraten kann es auch momentan zum Heben des Schwanzes kommen, aber nie in der charakteristischen Art und nie dauernd; die Männchen lassen sich überhaupt nicht treiben, sondern richten sich am Verfolger auf und beginnen den Kampf.

2. Die feminierten Tiere - Ratten wie Meerschweinchen - zeigen ferner den "Abwehrreflex". Dieser Reflex ist eine ausgeprägt weibliche Erscheinung von grosser Zweckmässigkeit. Er besteht im Hochheben eines Hinterfusses und in abstreifenden Bewegungen desselben, wodurch der Aufsprung des nachdrängenden Männchens verhindert wird. Er schützt das nichtbrünstige Weibchen vor unnützer sexueller Belästigung und vor unfruchtbarem

1) Vgl. Stein a, ch, Physiol. Zentralbl. 1910 S. 566. 
Coitus. Nur sehr rabiate Männchen sind imstande, trotz dieser heftigen Abwehr den Akt zu erzwingen.

3. In den vorstehenden Beobachtungen spiegelt sich auch schon das wesentlichste und untrüglichste Zeichen für die sexuelle Disposition der feminierten Tiere; siesind den normalen Männchen nicht indifferent wie Kastraten, sondern erwecken starken Geschlechtstrieb, werden als Weibchen agnosziert und behandelt.

Diesbezügliche Versuche dürfen nur mit fremden Männchen und nicht mit den mit aufgezogenen, daher mehr gleichgültigen Brüdern angestellt werden. Die Probiermännchen müssen ferner, wie bei allen genauen Prüfungen des Sexualtriebes, einige Zeit isoliert worden sein. Setzt man in den geräumigen Käfig eines solchen Männchens ein feminiertes Tier und einen Kastraten, so werden zwar zunächst beide verfolgt und beschnuppert, aber sehr bald nimmt das Männchen die Spur des feminierten Tieres auf, treibt es in grosser Erregung vor sich hin und versucht unablässig den Aufsprung, der ihm trotz der Abwehr zuweilen gelingt. Fin brünstiges Weibchen, bei welchem der starke Reiz der vaginalen Sekretion hinzukommt, wird natürlich bevorzugt. Hingegen erweist sich die Wirkung eines nichtbrünstigen Weibchens und eines feminierten Tieres auf das normale Männchen ganz gleichwertig. Die operierten Tiere haben weibliches Wesen, weibliche Stimmung erworben.

Die Ergebnisse unserer früheren Versuchsreihen, welche demonstrieren, wie beim männlich angelegten Individum sich Mamma und Brustwarze ausbilden, WachstumundGestaltung des Skeletts und des ganzen Körpers weiblichen Charakter annehmen, und endlich die Art der Behaarung und der lokale Fettansatz den weiblichen Typus vollenden, haben durch die obigen physiologischen Beobachtungen die wünschenswerte Ergänzung gefunden. Weder die sekundären somatischen noch die sekundären psychischen Geschlechtsmerkmale sind unwandelbar ab ovo vorausbestimmt. Sie können transformiert bzw. umgestimmt werden. Je früher der Austausch der Pubertätsdrüsen erfolgt, desto umfassender wird ihr fundamentaler Einfluss auf die neue Geschlechtsrichtung des Individums. 
8. Anhang.

\section{Das Auftreten von heterologen Geschlechtscharakteren im individuellen Leben.}

Die vorliegende Untersuchung hat zwei Grundtatsachen erhoben, welchen man auch bei Erklärung des Entstehens heterologer Merkmale im Verlaufe des individuellen Lebens wird Rechnung tragen müssen.

Erstens ist die Möglichkeit dargetan, nach Ausschaltung der Funktion der homologen Pubertätsdrüse und Einschaltung der heterologen Pubertätsdrüse die heterologen Merkmale zum Wachstum und zur vollen Ausbildung zu bringen, und dadurch eine Umwandlung des ursprünglichen Geschlechtscharakters hervorzurufen; und zwar kann diese Wirkung zum Teil auch eintreten, wenn nicht die ganzen implantierten Drüsen, sondern bloss Reste derselben funktionstüchtig bleiben. Zweitens ist die doppelte Tätigkeit der Pubertätszellen erwiesen: sie fördern nicht nur die Ausbildung aller homologen Sexuszeichen, sondern sie hemmen auch das Wachstum gewisser heterologer Merkmale, was bisher bekanntlich ein theoretisches Postulat war [Herbst ${ }^{1}$.

Was hier auf experimentellem Wege durchgesetzt worden ist, kann sich auch in der Natur ereignen. Man darf aber nie aus dem Auge verlieren, dass die bekannten und oft beschriebenen Fälle bei Mensch und Tier relativ seltene Ausnahmen von der Regel darstellen. Für diese Ausnabmen, bei welchen nach Erkrankung oder sogenanntem Funktionsausfall der Keimdrüsen einzelne oder mehrere heterologe Charaktere entstanden sind, wird man, wie schon oben bemerkt, annehmen müssen, dass keine vollständige und durchgreifende Differenzierung der Keimstockanlage statthatte, dass also in der betreffenden Keimdrüse sich männliche wie weibliche Pubertätszellen entwickelt finden.

Unter dieser Voraussetzung werden z. B. beim männlichen Individuum die überwiegenden männlichen Pubertätszellen zunächst die Wirksamkeit der vorhandenen weiblichen Pubertätszellen hemmen, und es tritt demgemäss rein der männliche Geschlechtscharakter mit allen seinen Attributen in die Erscheinung. Es kann nun vorkommen, dass durch Erkrankung oder durch vorzeitige Schwächung die männlichen Pubertätszellen leiden, und dass infolgedessen nicht

1) Formative Reize in der tierischen Ontogenese. 1901. 
allein die homologen Charaktere an Kraftfülle einbüssen, sondern auch die vorhandenen weiblichen Pubertätszellen durch Fortfall der Hemmung "aktiviert" werden und jetzt ihren fördernden Einfluss auf einzelne oder mehrere heterologe Merkmale betätigen. So kann, um an die üblichen Beispiele zu erinnern, bei dem Mann eine Mamma, bei der Frau ein Bart, beim Cervidenweibchen Geweih, bei der Henne Hahnenfedrigkeit entstehen.

Je früher im individuellen Leben die Schwächung der ursprünglich herrschenden Pubertätszellen einsetzt, desto ausgeprägter werden sich die sekundär-hermaphroditischen Erscheinungen gestalten; je später sie erfolgt, desto seltener, vereinzelter oder unmerklicher wird eine Aktivierung von heterologen Merkmalen zur Geltung kommen. Derselbe Vorgang, der das eine Mal zur Wandlung der somatischen Sexuszeichen führt, kann das andere Mal auch die Umstimmung des psychischen Sexualcharakters bewirken.

Es ist nicht notwendig, die hermaphroditische oder bisexuelle Anlage der Keimdrüsen zum allgemeinen biologischen Prinzip zu erheben, wie dies wiederholt geschehen ${ }^{1}$ ). Mit dieser Annahme steht auch die relative Seltenheit der Fälle in scharfem Widerspruch. So sieht man z. B. in der Menopause doch nur ausnahmsweise männliche Charaktere auftreten, die Unzahl der Frauen bleibt frauenhaft bis zum Tode. Es würde sich eher empfehlen, bis zur Differenzierung des Keimstocks von einem asexuellen Zustand zu sprechen.

Wenn es gelänge, männliche und weibliche (interstitielle) Pubertätszellen histologisch, färbetechnisch zu unterscheiden, so könnte man an Stelle obiger Hypothese den Nachweis setzen. Ich habe hier die Frage im Anschluss an meine Versuchsergebnisse nur flüchtig berührt und hoffe später weitere Beiträge zu liefern und auf das ganze Problem noch ausführlicher zurückzukommen.

Herrn Hofrat Professor R. von Wettstein danke irh auch hier verbindlichst für die Förderung dieser Arbeiten durch Überlassung eines staatlichen: Platzes an der biologischen Versuchsanstalt in Wien.

1) Vgl. hierzu die vortreffliche Darstellung der bezüglichen Frage und Literatur in A. Biedl's "Innere Sekretion". 


\section{Tafel erk lär ung.}

\section{Tafel III.}

Entwicklung des Mamma-Apparates bei Männchen nach der OvarienImplantation. Unterschied zwischen dem normalen und feminierten Männchen.

Fig. 1. Normales erwachsenes Meerschweinchen-Männchen; $2 / 3$ der natürlichen Grösse. $B w$ : Brustwarze (natürl. Grösse). Wh: Warzenhof. $G P$ : Glans penis.

Fig. 2. Feminiertes Meerschweinchen-Männchen; im Alter von drei Wochen operiert; 6 Monate alt; ${ }^{2 / 3}$ der natürlichen Grösse. $O v$ : Vorwölbungen, welche den Sitz der subcutan implantierten Ovarien anzeigen. $B w$ : Brustwarze (natürl. Grösse). Wh: Warzenhof. $G P$ : Glans penis.

\section{Tafel IV.}

Entwicklung des Mamma-Apparates beim Männchen nach OvarienImplantation (Photogram m).

Feminiertes Meerschweinchen-Männchen; im Alter von 3 Wochen operiert; im Alter von 5 Monaten photographiert. $O v$ : Vorwölbungen, welche den Sitz der subcutan implantierten Ovarien anzeigen. $B w:$ Brustwarze. $W h$ : Warzenhof. $E$ : Penis.

$$
\text { Tafel V-VII. }
$$

Röntgenaufnahmen von Ratten, welche die Femination der Männchen in bezug auf Wachstum, Dimensionierung und Gestaltung des ganzen Körpers bzw. Skeletts demonstrieren. Unterschied zwischen normalen und kastrierten Männchen einerseits und feminierten Männchen andererseits; jede Gruppe aus Tieren desselben Wurfes bestehend.

Die Bilder sind unter konstanten Bedingungen aufgenommen; zum Zwecke der Reproduktion verkleinert. Die vergleichenden Knochenmessungen auf Grund der Originalplatten siehe Tabelle VII.

Taf. V: Gruppe 1. Normales Männchen und normales Weibchen (Schwester) aus einem 15 Monate alten Wurf; Beispiel für den typischen Unterschied zwischen beiden Geschlechtern.

Taf. VI: Gruppe 2. Normales Männchen und feminierter Bruder aus einem 9 Monate alten Wurf.

Taf. VII: Gruppe 3. Männlicher Frühkastrat und feminierter Bruder aus einem 8 Monate alten Wurf.

\section{Tafel VIII.}

Photogramme eines Meerschweinchenpaares und zweier Rattenpaare. Je ein Paar auf derselben Platte aufgenommen. Jedes Paar stammt aus einem Wurf und besteht aus dem feminierten Männchen und seinem normalen Bruder. Gesamteindruck der feminierten Tiere, bzw. des willkürlich erzeugten weiblichen Geschlechtscharakters. Kontrast im Aussehen zwischen den feminierten und normalen Männchen. Sämtliche Tiere befinden sich derzeit noch am Leben. 
108 E. Steinach: Willkürlíche Umwandlung von Säugetier-Männchen etc.

Paar 1. Meerschweinchen im Alter von 5 Monaten photographiert (die Implantationsstellen und der ausgebildete Mamma-Apparat des feminierten Tieres siehe auf Tafel IV). $F:$ Feminiertes Männchen. $M:$ Normaler Brader. Die weisse Trennungslinie ist erst bei der Reproduktion entstanden, und zwar dadurch, dass mit Rücksicht auf das Tafelformat die Distanz zwischen den Einzelbildern veringert werden musste.

Paar 2. Ratten im Alter von $9^{1 / 2}$ Monaten photographiert (Momentaufnahme). $F$ : Feminiertes Männchen. $M$ : Normaler Bruder.

Paar 3. Ratten im Alter von 8 Monaten photographiert (narkotisiert und gleichmässig aufgebunden). Röntgenaufnahme dieses Paares siehe Tafel VI Gruppe 2. $F$ : Feminiertes Männchen. $M$ : Normaler Bruder. 
Fig. 1.
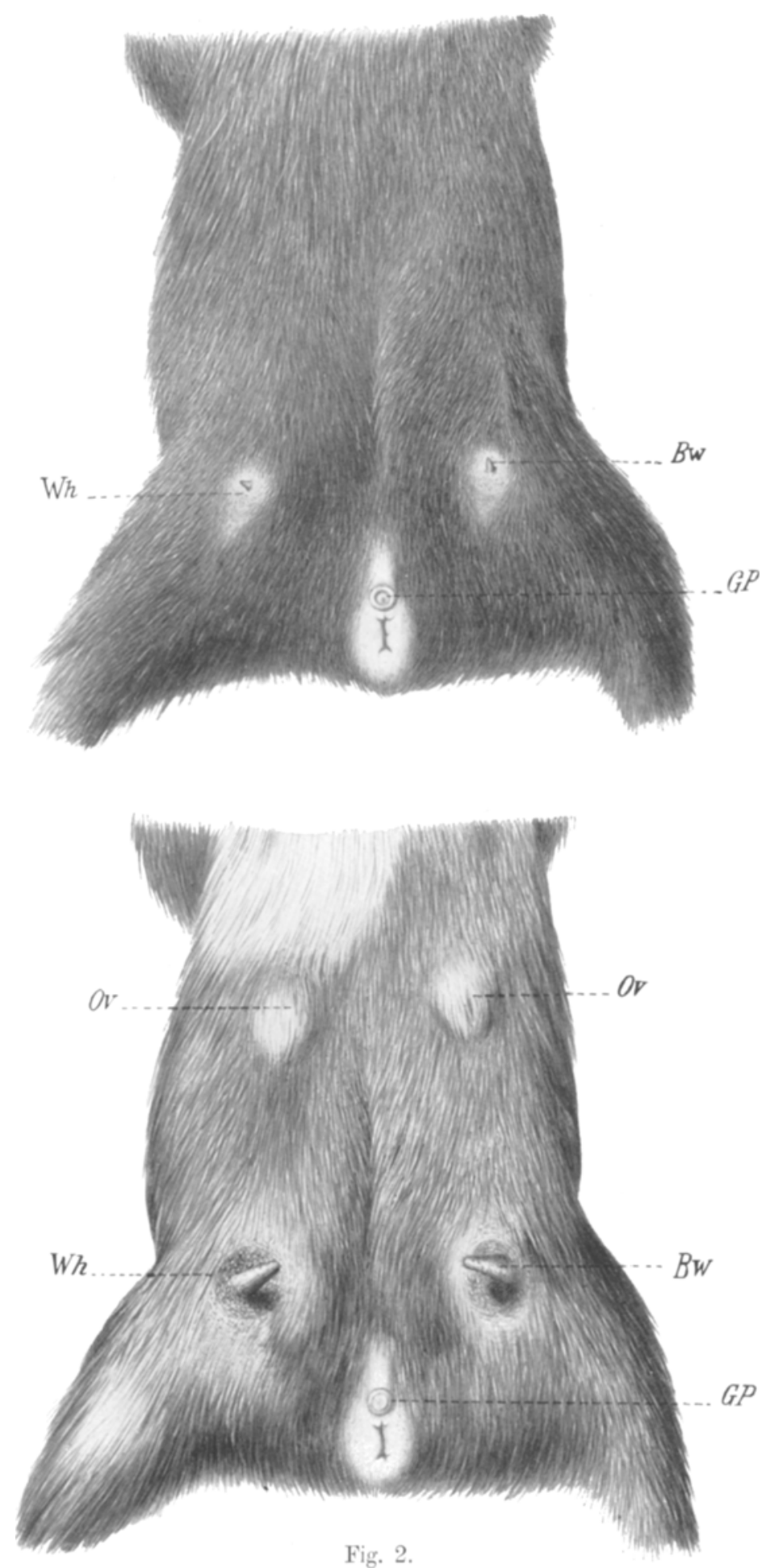

Verlag von Martin Hager, Bonn. 
Pflüger’s Archiv f. d. ges. Physiologie. Bd. 144.

Tafel IV.

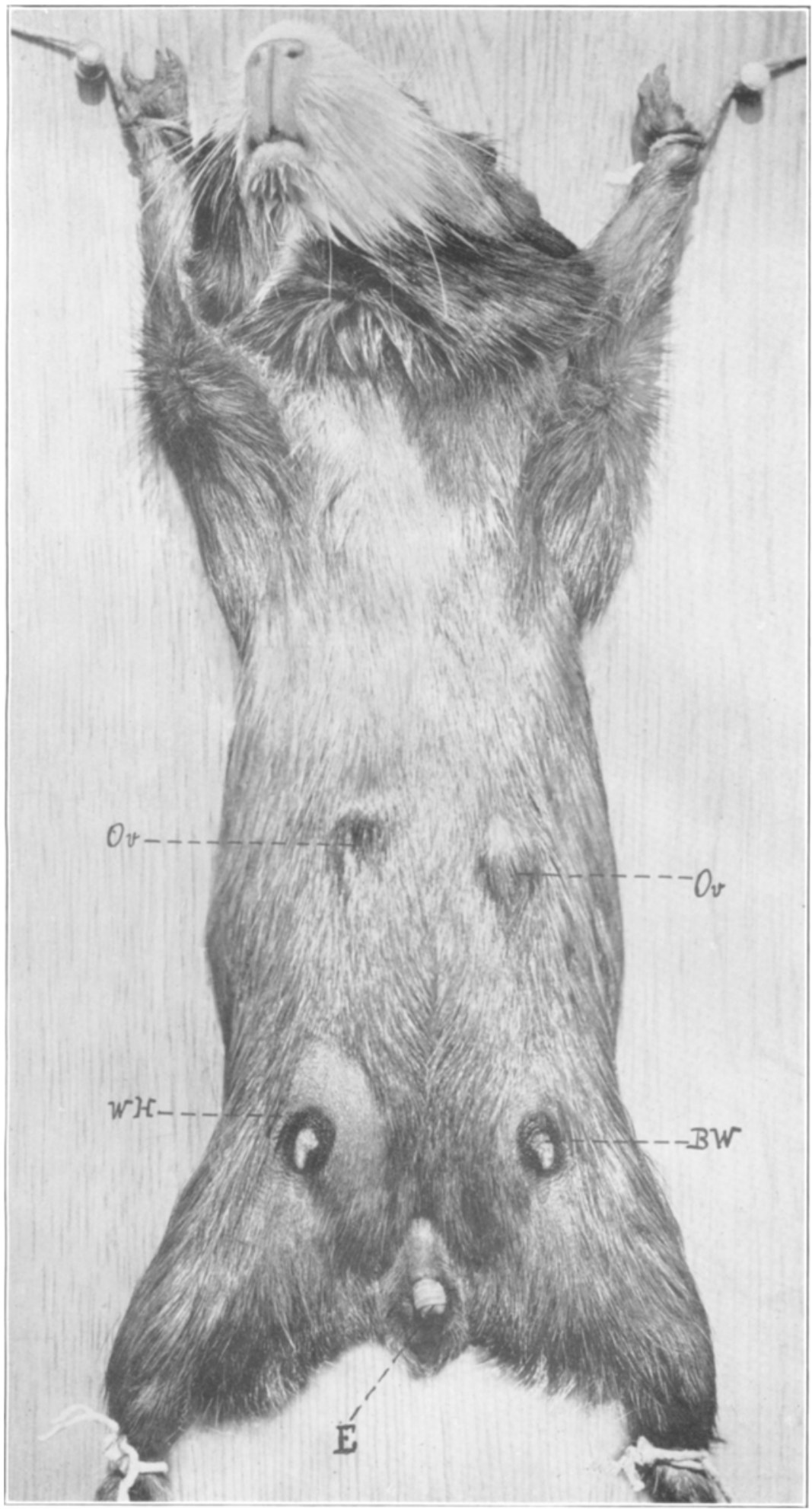


Fllugers Archiv fid ges. Physidogege. Bd, 144 .

of nomal

P narmal (Schirestan)

Gro 1
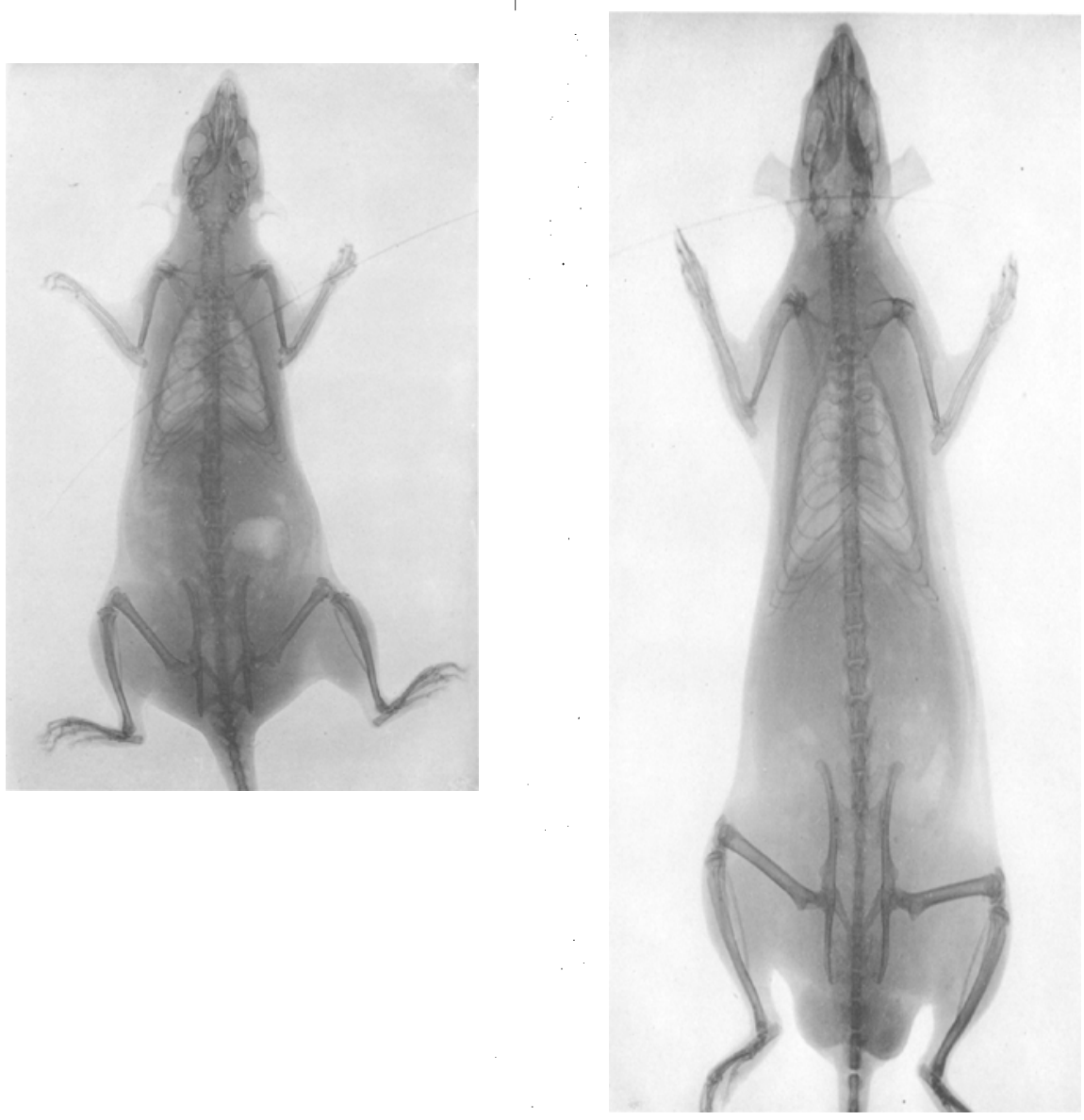

Fon Martin Hager, Boun 

feningiarter Broder

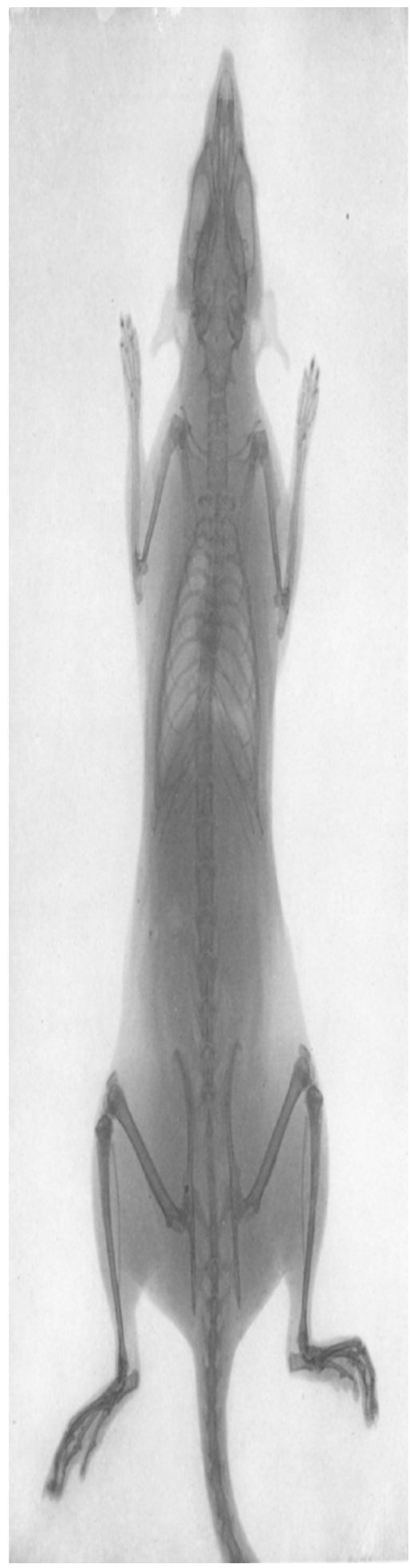

Gruppe

\section{त) Prublkastrat}

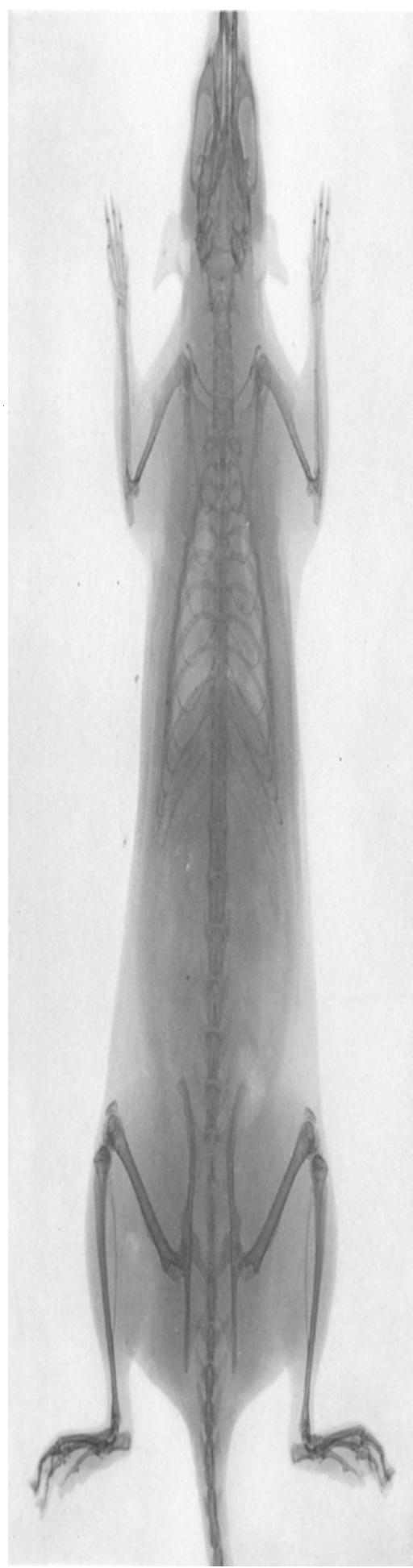

Terlag sou Kartin Hager, Bonn. 
Pflüger's Archiv f. d. ges. Physiologie. Bd. 144.
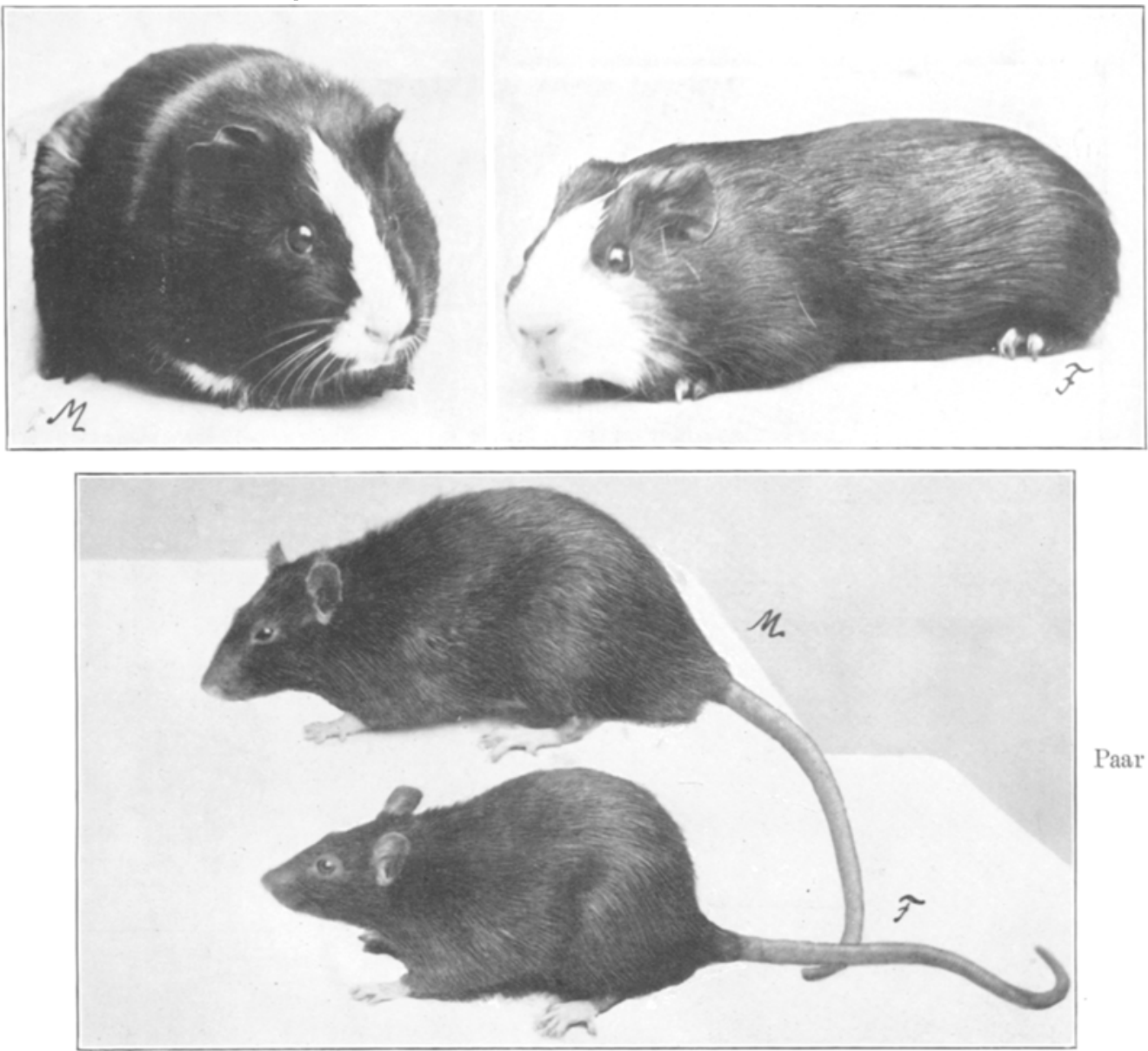

Paar 2.

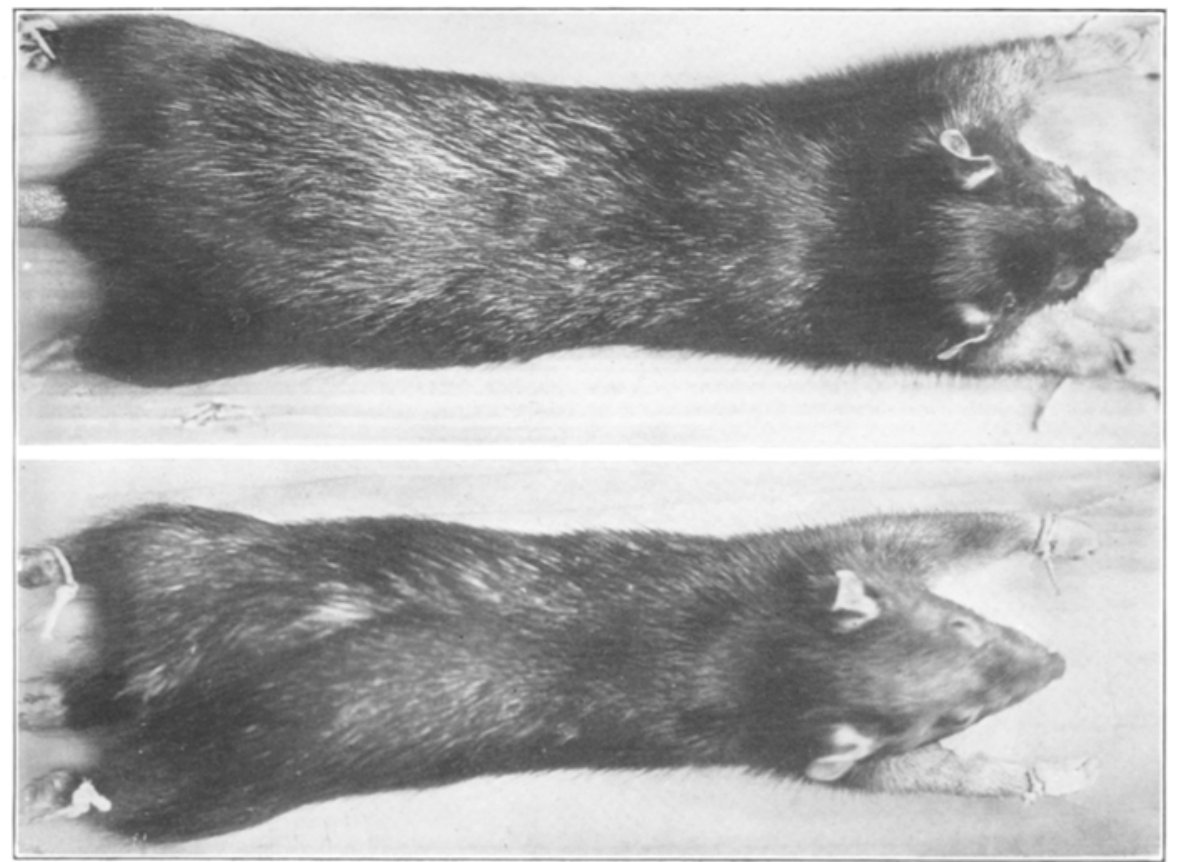

Paar 3. 